\title{
Uncertainty Quantification Techniques for Sensor Calibration Monitoring in Nuclear Power Plants
}
P Ramuhalli
BG Braatz
G Lin
JB Coble
SL Crawford
BD Shumaker
A Konomi
HM Hashemian

September 2013

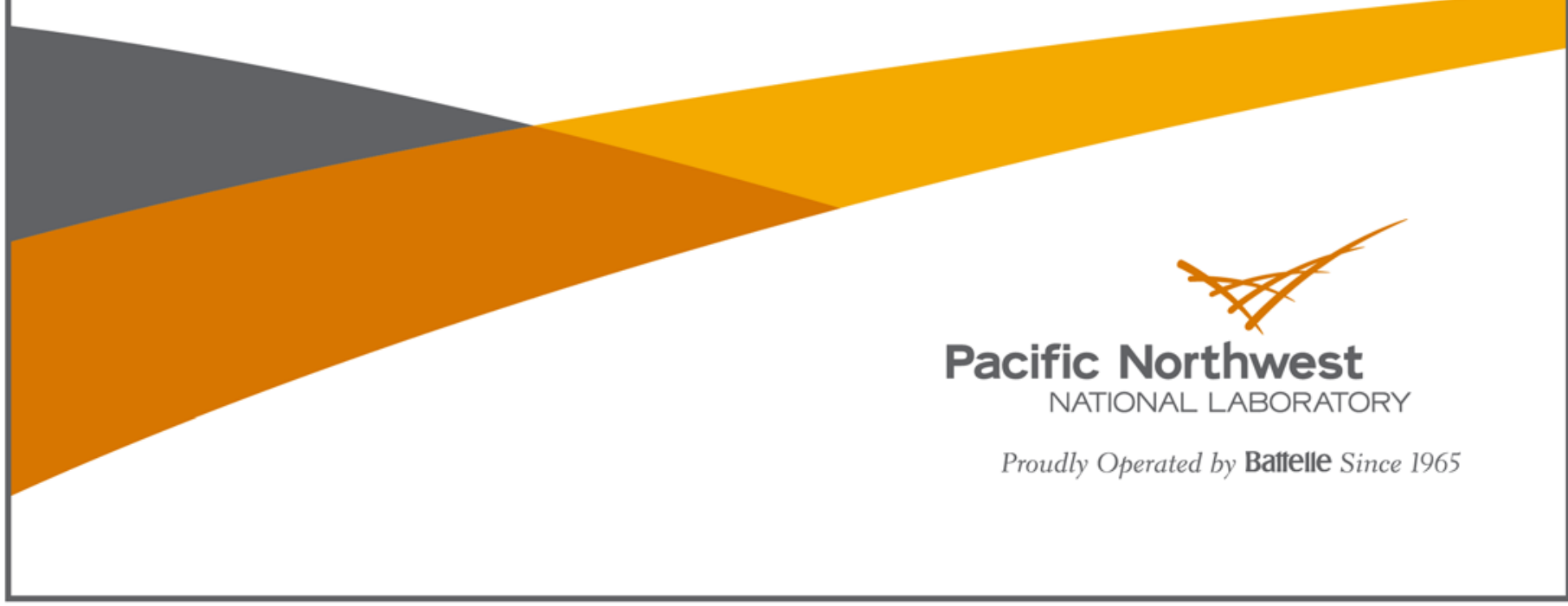




\title{
DISCLAIMER
}

This report was prepared as an account of work sponsored by an agency of the United States Government. Neither the United States Government nor any agency thereof, nor Battelle Memorial Institute, nor any of their employees, makes any warranty, express or implied, or assumes any legal liability or responsibility for the accuracy, completeness, or usefulness of any information, apparatus, product, or process disclosed, or represents that its use would not infringe privately owned rights. Reference herein to any specific commercial product, process, or service by trade name, trademark, manufacturer, or otherwise does not necessarily constitute or imply its endorsement, recommendation, or favoring by the United States Government or any agency thereof, or Battelle Memorial Institute. The views and opinions of authors expressed herein do not necessarily state or reflect those of the United States Government or any agency thereof.

\author{
PACIFIC NORTHWEST NATIONAL LABORATORY \\ operated by \\ BATTELLE \\ for the \\ UNITED STATES DEPARTMENT OF ENERGY \\ under Contract DE-AC05-76RL01830
}

Printed in the United States of America
Available to DOE and DOE contractors from the Office of Scientific and Technical Information,
P.O. Box 62, Oak Ridge, TN 37831-0062;
ph: (865) 576-8401
fax: $(865) 576-5728$
email: reports@adonis.osti.gov

\author{
Available to the public from the National Technical Information Service, \\ U.S. Department of Commerce, 5285 Port Royal Rd., Springfield, VA 22161 \\ ph: (800) 553-6847 \\ fax: (703) 605-6900 \\ email: orders@ntis.fedworld.gov \\ online ordering: http://www.ntis.gov/ordering.htm
}

This document was printed on recycled paper. 


\title{
Uncertainty Quantification Techniques for Sensor Calibration Monitoring in Nuclear Power Plants
}

\author{
P Ramuhalli \\ BG Braatz \\ G Lin \\ JB Coble ${ }^{(a)}$ \\ SL Crawford \\ BD Shumaker ${ }^{(b)}$ \\ B Konomi \\ HM Hashemian $^{(b)}$
}

September 2013

Prepared for

the U.S. Department of Energy

under Contract DE-AC05-76RL01830

Pacific Northwest National Laboratory

Richland, Washington 99352

(a) Formerly at Pacific Northwest National Laboratory

Presently at University of Tennessee, Knoxville, Tennessee 37923

(b) Analysis \& Measurement Services Corporation

Knoxville, Tennessee 37923 



\section{Executive Summary}

Safe, efficient, and economic operation of nuclear systems (nuclear power plants, fuel fabrication and storage, used fuel processing, etc.) relies on accurate and reliable measurements. Newer types of sensors, and sensors to monitor non-traditional parameters, are expected in next-generation nuclear power plant (NPP) and fuel-cycle environments. A number of factors (besides changes in the monitored variable) affect the measured signals, resulting in effects such as signal drift and response time changes, requiring techniques to distinguish between signal changes from plant or subsystem performance deviations and those from sensor or instrumentation issues. Advanced algorithms that continuously monitor sensor responses can address this issue and facilitate automated monitoring and control of plant and subsystem performance.

Currently, periodic sensor recalibration is performed to avoid problems with signal drift and sensor performance degradation. Periodic sensor calibration involves (1) isolating the sensor from the system, (2) applying an artificial load and recording the result, and (3) comparing this "As Found" result with the recorded "As Left" condition from the previous recalibration to evaluate the drift at several input values in the range of the sensor. If the sensor output is found to have drifted from the previous condition, then the sensor is adjusted to meet the prescribed "As Left" tolerances. However, this approach is expensive and time-consuming, and unnecessary maintenance actions can potentially damage sensors and sensing lines. Online monitoring (OLM) can help mitigate many of these issues, while providing a more frequent assessment of calibration and signal validation. However, widespread utilization of traditional OLM approaches is lacking with the need to better quantify OLM uncertainty a key factor in this.

Sources of uncertainty in OLM can be roughly categorized as (1) process noise, (2) measurement uncertainty, (3) electronic noise, and (4) modeling uncertainty. Approaches to uncertainty quantification (UQ) that are data-driven may be capable of providing estimates of uncertainty that are time-varying as the quantities being measured vary with time. Such a capability provides the option of adjusting acceptance criteria and, potentially, setpoints in a time-varying fashion to meet the needs of the nuclear power system.

A Gaussian Process (GP) model is proposed in this study for addressing the UQ issue. The advantage of this approach is the ability to account for spatial and temporal correlations among the sensor measurements that are used in OLM. The GP model, as proposed, may be considered an extension of a commonly used OLM model and, therefore, the hypothesis is that the UQ methodology may be readily extended to accommodate commonly used OLM models.

Two approaches were taken for generating the data sets needed for evaluating the proposed model. Experimental data was acquired using an instrumented flow loop, with varying test conditions. In addition, a simulation model of a flow loop was generated. The simulation model serves two purposes. First, data may be generated from the simulation model that represent conditions the experimental flow loop may not be able to achieve. Second, the use of a model can provide insights into physical relationships between measurements from sensors at different locations. These insights can be used to derive new algorithms for OLM, as well as develop methods for generating virtual sensors. Although analysis of data generated from the simulation models is continuing, the models themselves are included for completeness. 
Initial assessment of the proposed UQ methodology using data from an instrumented flow loop indicates the feasibility of generating error bounds on measurement data that are time-dependent. However, the assessment uses only steady-state data from normal conditions (i.e., no sensor faults are present) and will need to be extended to include data from sensor faults and non-steady-state conditions.

Ongoing work includes completing the assessment of the GP approach to data-driven uncertainty quantification using additional experimental data sets, as well as data covering a wider range of conditions (including data from sensor faults and non-steady-state conditions). In addition, the applicability of the proposed approach to a wider set of existing OLM models will need to be examined. Ongoing work with regard to the simulation models includes incorporating the collected measurement data parameters into the model to evaluate or validate the model results. Additionally, the variable flow model will be modified to ensure that it is a robust model of the flow loop, prior to using the simulation data for further evaluating the UQ methodology.

The research being conducted under this "Recalibration Methodology" project (part of the Nuclear Energy Enabling Technologies Advanced Sensors and Instrumentation - NEET/ASI - Program) supports the needs of several U.S. Department of Energy's Office of Nuclear Energy programs, including Light Water Reactor Sustainability (LWRS), Small Modular Reactor (SMR) R\&D, Advanced Reactor Concepts (ARC), Materials Protection, Accounting, and Control Technologies (MPACT), Fuel Cycle Research and Development (FCRD), and Next Generation Nuclear Plant (NGNP) programs, through the development of advanced algorithms for monitoring sensor/system performance and enabling the use of plant data to derive information that currently cannot be measured. These advances are expected to improve the safety and reliability of current and planned nuclear power systems as a result of higher accuracies and increased reliability of sensors used to monitor key parameters. 


\section{Acknowledgments}

The authors gratefully acknowledge Kay Hass for her invaluable assistance in the technical editing and formatting of this report. 



\section{Acronyms and Abbreviations}

AAKR

ASI

CSA

EPRI

EULM

GP

gPC

IAEA

ICA

NEET

NPP

NRC

OLM

PC

RTD

SMR

SPRT

SPSS

UQ
Auto-Associative Kernel Regression

Advanced Sensors and Instrumentation (program)

channel statistical accuracy

Electric Power Research Institute

error uncertainty limit monitoring

Gaussian Process

generalized polynomial chaos

International Atomic Energy Agency

independent component analysis

Nuclear Energy Enabling Technologies

nuclear power plant

U.S. Nuclear Regulatory Commission

online monitoring

polynomial chaos

resistance temperature detector

small modular reactor

sequential probability ratio test

Stochastic Parameter Simulation System

uncertainty quantification 



\section{Contents}

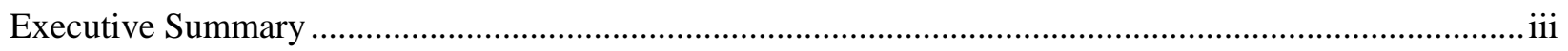

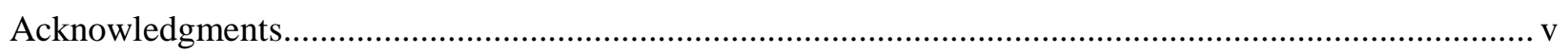

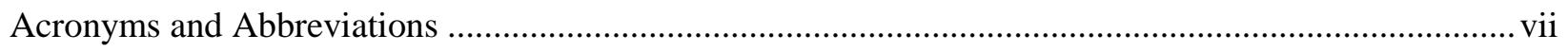

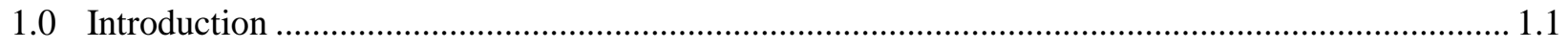

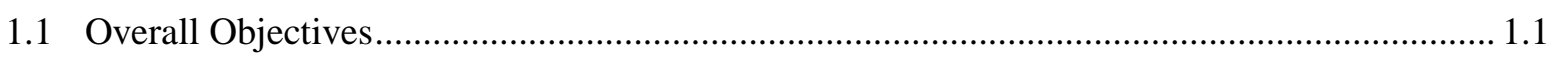

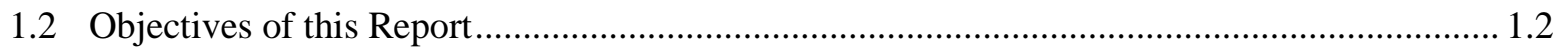

1.3 Motivation and Potential Benefits................................................................................... 1.2

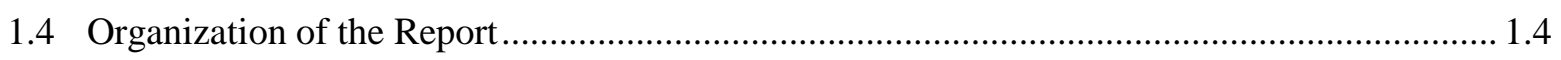

2.0 Background: Sensor Calibration and Online Monitoring ............................................................. 2.1

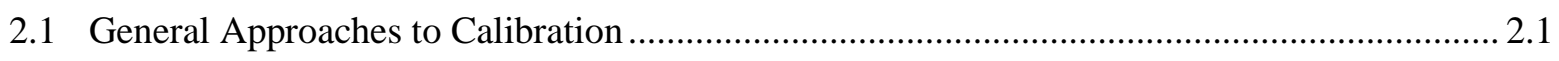

2.2 Online Monitoring for Sensor Calibration Interval Extension ................................................ 2.2

2.3 Current Approaches to Uncertainty for OLM ..................................................................... 2.3

3.0 A Data-Driven Approach to Uncertainty Quantification.............................................................. 3.1

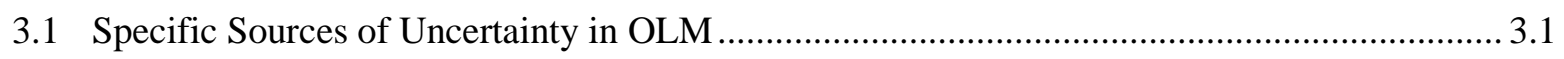

3.2 General Principles of Uncertainty Quantification .................................................................. 3.2

3.3 Uncertainty Analysis Assumptions ................................................................................. 3.3

3.4 Uncertainty Quantification Methods for Uncertainty Estimation in Online Sensor

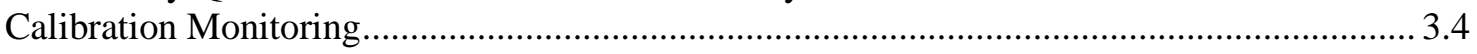

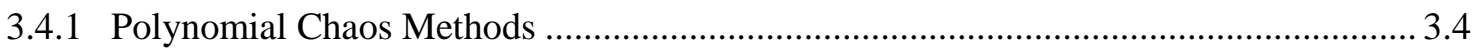

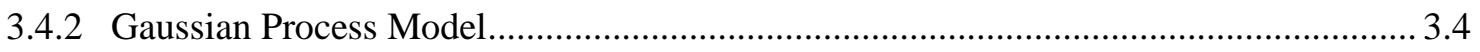

3.4.3 Estimates of Noise on Sensors and UQ Methods for De-noising................................. 3.6

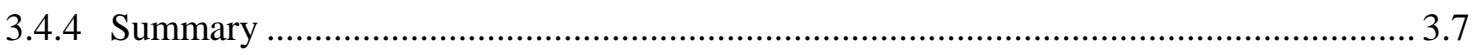

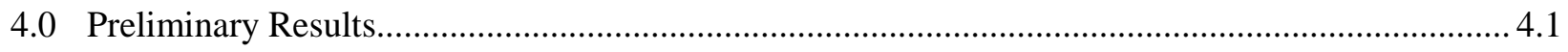

4.1 Experimental Data for Evaluation of UQ Methods........................................................... 4.1

4.1.1 Flow Loop Configuration....................................................................................... 4.1

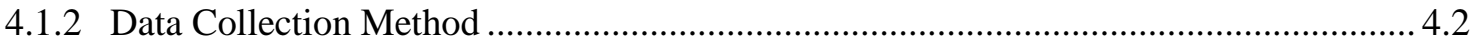

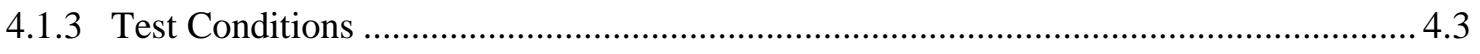

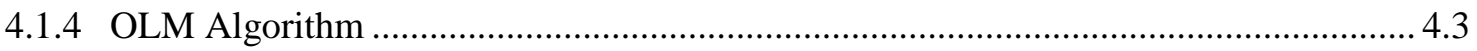

4.2 Uncertainty Analysis and Quantification .......................................................................... 4.5

4.3 Simulation Models (Flow Loop Model with Counter Flow Heat Exchanger) ....................... 4.10

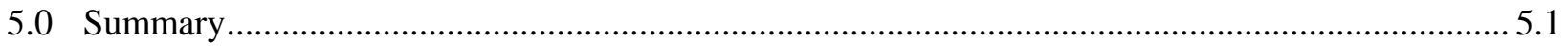

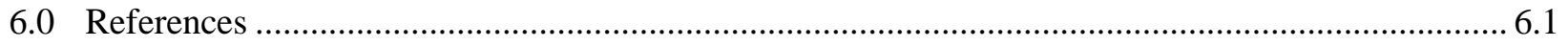




\section{Figures}

4.1 Flow Loop Piping and Instrumentation Diagram.......................................................................... 4.2

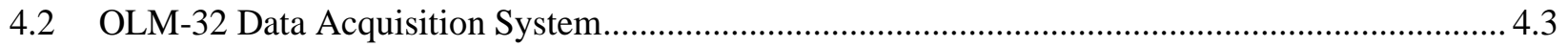

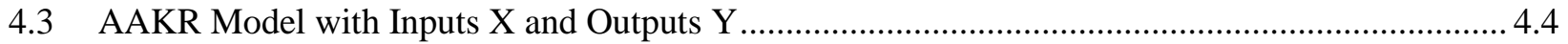

4.4 Snapshot of Predictions for All Sensor Data, Assuming a Single Input Variable .......................... 4.6

4.5 Predicted Response Surfaces at a Single Time Instant, Assuming Two Input Variables ................ 4.7

4.6 Flow Loop Control Model with Constant Flow ......................................................................... 4.11

4.7 Flow Loop Details with Heater on the Left, Heat Exchanger on the Right, and Process Noise Added at the Poisition Noted by the Arrow …..................................................................... 4.12

4.8 Input Setpoint Temperature on the Left and the Output Temperature on the Right ..................... 4.12

4.9 Temperatures at Different Locations in the Flow Loop, Scope 7 on the Left and Scope 1 on

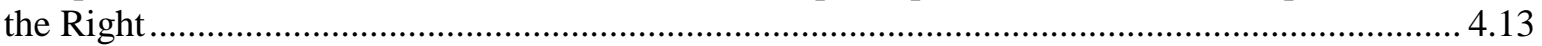

4.10 Flow Loop Model with Variable Temperature and Flow............................................................ 4.13

4.11 Flow Loop Details with Heater, Pump Control, Heat Exchanger, and Multiple Sensors .............. 4.14

\section{Tables}

4.1 Constant Flow Model Inputs, Assumptions, and Outputs

4.10 


\subsection{Introduction}

Safe, efficient, and economic operation of nuclear systems (nuclear power plants, fuel fabrication and storage, used fuel processing, etc.) relies on accurate and reliable measurements. Newer types of sensors, and sensors to monitor non-traditional parameters, are expected in next-generation nuclear power plant (NPP) and fuel-cycle environments. During operation, components of nuclear systems, including sensors, may degrade from age, environmental exposure, and even maintenance interventions. These (and other) factors (besides changes in the monitored variable) affect the measured signals, resulting in effects such as signal drift and response time changes. There is a need to distinguish between signal changes from plant or subsystem performance deviations and those from sensor or instrumentation issues. Advanced algorithms that continuously monitor sensor responses can address this issue and facilitate automated monitoring and control of plant and subsystem performance.

Currently, periodic sensor recalibration is performed to avoid problems with signal drift and sensor performance degradation. However, this approach is expensive and time consuming, and unnecessary maintenance actions can potentially damage sensors and sensing lines. The use of online monitoring (OLM) can help mitigate many of these issues, while providing a more frequent assessment of calibration and signal validation. OLM is a non-invasive approach to assess measurement accuracy and component condition. OLM systems generally use models of the plant (or subsystems thereof) to estimate the expected sensor values for a group of redundant or related measurements (Hashemian and Jiang 2009). If an anomaly is detected, diagnostic routines are used to determine if it is the result of a sensor fault or a change in the condition of the process/component and to isolate the location of the fault.

Widespread utilization of traditional OLM approaches is lacking, and application of signal validation and virtual sensors has not yet entered the commercial space for nuclear systems. Previously, several technical gaps were identified relative to the deployment of OLM in nuclear power systems (Coble et al. 2012). These technical gaps will need to be addressed to provide the technical and regulatory bases for wider adoption of these technologies. As the nation looks to the next generation of nuclear systems (reactor technologies, fuels, and fuel cycle), a number of other factors are likely to also slow the adoption of existing OLM methodologies, including its applicability to non-traditional sensor systems and the increased use of digital instrumentation and controls and wireless technologies. Underpinning all of these is the need to better quantify OLM uncertainty to: (1) support the regulatory basis for OLM-based calibration assessment, (2) provide the high confidence levels needed for signal validation, (3) provide virtual sensor estimates with meaningful confidence, and (4) evaluate the efficacy of these techniques for new sensors and data acquisition systems.

\subsection{Overall Objectives}

The overall objective of this research is to develop the next generation of online monitoring technologies for sensor calibration interval extension and signal validation in operating and new reactors. This "Recalibration Methodology” research project (part of the Nuclear Energy Enabling Technologies Advanced Sensors and Instrumentation - NEET/ASI - Program) supports the needs of several U.S. Department of Energy's Office of Nuclear Energy programs, including Light Water Reactor Sustainability (LWRS), Small Modular Reactor (SMR) R\&D, Advanced Reactor Concepts (ARC), Materials Protection, Accounting, and Control Technologies (MPACT), Fuel Cycle Research and Development (FCRD), and Next Generation Nuclear Plant (NGNP) programs, through the development 
of advanced algorithms for monitoring sensor/system performance and enabling the use of plant data to derive information that currently cannot be measured. These advances are expected to improve the safety and reliability of current and planned nuclear power systems as a result of higher accuracies and increased reliability of sensors used to monitor key parameters. Over the course of this project, the goal is to assess methods for improved uncertainty quantification in online monitoring, and use the results to develop robust virtual sensor technology and formal methods for signal validation.

\subsection{Objectives of this Report}

The focus of this report is on documenting the outcomes of the first phase of R\&D under this project, which addressed approaches to uncertainty quantification (UQ) in online monitoring that are data-driven, and can therefore adjust estimates of uncertainty as measurement conditions change. Such data-driven approaches to UQ are necessary to address changing plant conditions, for example, as nuclear power plants experience transients, or as next-generation small modular reactors (SMR) operate in loadfollowing conditions.

Several sources of uncertainty contribute to the overall OLM uncertainty, including process noise, measurement and electronic noise, and modeling errors and uncertainties; these sources need to be accounted for in a systematic manner that can be applied to a wide variety of modeling methods. The UQ results will impact the selection of appropriate acceptance criteria for anomaly detection that help discriminate between instrumentation failure and plant performance degradation. This is due to the need to incorporate the overall uncertainty in an OLM system into the fault detection routine to provide a prescribed level of confidence that the sensor is operating within specifications and that anomalies in plant operation can be identified. This issue is exacerbated when considering systems (such as SMRs) where the monitoring and UQ may need to be performed under conditions (such as load-following) not normally encountered in current light water reactors.

Research to address this specific gap will support several needs of the U.S. nuclear power industry, including improved monitoring to achieve higher accuracy and reliability of measured parameters over longer operational periods and reduced human errors.

\subsection{Motivation and Potential Benefits}

The current calibration procedure, referred to as "Conventional Calibration," "Manual Calibration," or "Technician Calibration," is effective and reliable and has been used in the industry since the inception of nuclear facilities. However, reviews of nearly 40 years of calibration data from nuclear plants have revealed that:

1. More than 90 percent of nuclear plant transmitters do not exceed their calibration acceptance criteria over a single fuel cycle (EPRI 2003).

2. Calibration activities can, on occasion, create problems that would not otherwise occur, such as inadvertent damage to transmitters caused by pressure surges during calibration, air/gas entrapped in the transmitter or its sensing line during the calibration, improper restoration of transmitters after calibration leaving isolation or equalizing valves in the wrong position (e.g., closed instead of open or vice versa), valve wear resulting in packing leaks, and valve seat leakage (EPRI 1998). 
Extending the periodic manual calibrations in NPPs by incorporating OLM affords several direct benefits (Hashemian 2011) including increased reliability, safety, and efficiency in the plant. OLM methods can also provide a significant financial savings, especially if outage duration is reduced. Similar benefits are expected with the use of OLM in the next generation of nuclear power plants that are being constructed or proposed in the United States. These include the Gen III+ and advanced reactor designs, including SMR designs. The anticipated harsher operating conditions (particularly in longer-term advanced designs), along with potential new sensor materials and designs, may lead to new sensor degradation modes and characteristics. These factors will impact the calibration burden on sensors. When combined with an extended refueling cycle (from $\sim 1.5$ years presently to $\sim 4-6$ years or longer as advanced reactors come online), the ability to ensure reliable operation and extend calibration intervals by monitoring the calibration performance online becomes increasingly important to maintain safe operating envelopes.

A previously published assessment (Coble et al. 2012) identified several technical gaps that preclude the widespread adoption of OLM for calibration monitoring. Of the gaps identified, better approaches to UQ were identified as important to both current approaches to OLM as well as in any future advanced methods for OLM. Research to address these gaps (and in particular, the gap associated with UQ) will support several goals of the Nuclear Energy Enabling Technologies (NEET) Advanced Sensors and Instrumentation (ASI) Program, ${ }^{(a)}$ including improved monitoring to achieve higher accuracy and reliability of measured parameters over longer operational periods. The NEET ASI Program also aims to reduce the dependence on humans for certain tasks in order to reduce human errors. Errors in calibration and re-installation during routine maintenance are known to damage otherwise healthy sensors. By targeting recalibration to only those sensors that require it, these maintenance-induced faults and failures can be greatly reduced.

The research to address these gaps will have an impact on a number of programs currently supported by the U.S. Department of Energy's Office of Nuclear Energy. This research would complete the science base needed to enable adoption of OLM for sensor calibration interval extension in U.S. nuclear power plants, hence enabling utilities to potentially apply for the license amendment necessary to make the shift away from scheduled calibration methodologies. This directly supports the needs of the Light Water Reactor Sustainability program.

SMRs and advanced reactors are expected to employ longer operating cycles between maintenance outages, as well as more highly automated control and information systems; these expectations will require a greater awareness of sensor performance, which will be provided by online calibration monitoring. In addition, the ability to monitor instrumentation performance provides a level of automation that can help improve economics of plant operation by reducing unnecessary maintenance.

Similar benefits are envisioned for instrumentation (planned or in-use) in fuel-cycle systems. Identifying sensor performance degradation in a timely fashion is necessary to improve confidence in operations at nuclear fuel-cycle facilities, and is of particular importance in measurement campaigns that generate data for verifying and validating new fuel designs.

(a) DOE. Integrated Research Plan for Advanced Sensors and Instrumentation. Draft August 2012. U.S. Department of Energy (DOE), Office of Nuclear Energy. 


\subsection{Organization of the Report}

Section 2 provides the necessary background to online monitoring, including the sensor calibration needs in NPPs, current practices and standards used, and an overview of online monitoring. Section 3 describes the sources of uncertainty in NPP measurements, and the current practice for uncertainty quantification in OLM. Section 4 describes the UQ methodology that is proposed. A description of the experimental setup for measurement data collection, and results of evaluating the methodology using this data are provided in Section 5. Finally, some conclusions about the path forward for calibration monitoring and calibration interval extension are given in Section 6. 


\subsection{Background: Sensor Calibration and Online Monitoring}

Several types of measurement instrumentation are used in nuclear power plants to ensure safety and optimize the economics of operation. Instruments are incorporated for measurements of temperature, pressure, flow, neutron flux, and water chemistry (e.g., pH and conductivity). Additional instrumentation may be incorporated to monitor and indicate the position or state of several mechanical devices such as control rods and valves and to monitor the speed of rotating machinery (IAEA 2011). A detailed description of sensors used currently in nuclear power, as well as new developments in sensing that may be applicable for nuclear power systems, is provided in a previous report (Coble et al. 2012) that also includes a detailed description of sensor calibration state-of-practice.

This section summarizes sensor calibration practices in the nuclear power industry, and describes a general overview of OLM along with current methods for uncertainty quantification in OLM.

\subsection{General Approaches to Calibration}

Current practice for calibration of sensors generally depends on the type of sensor. For pressure, level, and flow transmitters, this task is accomplished using a pressure source, such as a dead weight tester, that is carried to the field. Each transmitter is isolated from the process and connected to the pressure source to be calibrated. A set of known pressure inputs (typically corresponding to 0, 25, 50, 75, and 100 percent of span) is applied and the output readings (usually measured in Volts or milliamperes) recorded. Sometimes the sensor is also given a decreasing set of pressure inputs $(75,50,25$, and 0 percent) to account for any hysteresis effect. This "As-Found" data is compared to the low and high As-Found acceptance limits. If the measured values are within the acceptance limits, no action is taken necessarily, although calibration technicians sometimes make adjustments to null deviations from perfect calibration even when the deviation is within acceptable limits. If a transmitter fails to meet its acceptance criteria, it is calibrated by adjusting the transmitter output for one or more known inputs iteratively until the input/output relationship meets the acceptance criteria. Typically, the acceptance limits after calibration are narrower than the "As-Found" limits to ensure that the transmitter calibration is adjusted closer to the desired output.

The calibration procedure for temperature instrumentation is different because temperature sensors cannot be calibrated in the normal sense unless they are removed from the plant and calibrated in a laboratory. Cross-calibration of temperature instrumentation is usually used to perform in-situ calibration (Hashemian 1990), and requires isothermal plant conditions during which all resistance temperature detector (RTDs) of interest are exposed to the same temperature. Under the isothermal condition, the readings of the RTDs are recorded and compared with each other to identify any outliers. An outlier RTD is then removed from the plant and replaced or calibrated in a laboratory. Newer approaches that use cross-calibration data collected during plant startup and/or shutdown periods on all RTDs at least three temperatures (e.g., 200, 250, and $300^{\circ} \mathrm{C}$ ) are also being examined (Hashemian 2006).

Thermocouples are almost never calibrated in-situ or even in a laboratory. A thermocouple that has been installed in a process cannot be recalibrated because new junctions can develop where there are large temperature gradients (where the thermocouple protrudes into the process environment). Although this is not usually a problem while the thermocouple is installed, it can cause large errors if the thermocouple is removed and calibrated in the laboratory. As such, when a thermocouple loses its calibration, it must be 
replaced. However, attempts are sometimes made to ensure that nuclear plant thermocouples that provide important temperature data to the control or safety systems are working properly. These attempts typically involve a procedure such as the cross calibration that was just explained. In doing this, thermocouples are often cross calibrated against RTDs, not against each other.

\subsection{Online Monitoring for Sensor Calibration Interval Extension}

Performance monitoring of NPP instrumentation has been an active area of research since the mid1980s (Deckert et al. 1983; Oh and No 1990; Ray and Luck 1991; Ikonomopoulos and van der Hagen 1997). Early research in signal validation for NPPs relied on direct or analytic measurement redundancy. However, additional methods that do not require redundant sensor measurements have been developed for application to both safety and non-safety sensors.

In 1995, Hashemian (1995a) outlined methods to test calibration of sensors during steady-state plant operation, including redundant channel averaging, process modeling, and comparison with calibrated reference channels. The results from pilot applications of OLM using a variety of methods for calibration monitoring show that common sensor faults, including sensor drift, sensor bias, stuck sensors, etc., can be reliably detected (Bickford et al. 2002; Davis et al. 2002; Hines and Davis 2005). The state of the art in OLM for sensor calibration interval extension was reviewed in the NUREG/CR-6895 series (Hines and Seibert 2006a; Hines et al. 2008a, b) and a recent International Atomic Energy Agency (IAEA) report (IAEA 2008).

Online calibration monitoring generally involves two steps: modeling the expected sensed values and evaluating the difference between the expected and actual behavior for faults. Several modeling methods have been proposed for evaluating sensor performance of redundant and non-redunant sensor groups (Coble et al. 2012). Modeling methods developed for non-redundant sensor groups require that the sensors in a single model contain related information (e.g., temperature and pressure of a gas), typically identified by linear correlations or a physical understanding of the measurement relationships. Redundant sensor groups can be paired with other, related measurements, and the full ensemble can be monitored through non-redundant sensor modeling methods. Generally, modeling methods developed for nonredundant sensor groups can also be applied to redundant sensor groups; however, the converse is not true. The difference between expected (modeled) and actual (measured) behavior, called the residual, characterizes system deviations from normal behavior and can be used to determine if the sensor or system is operating in an abnormal state. Fault detection methods commonly applied to residuals for calibration monitoring include simple thresholding, error uncertainty limit monitoring (EULM) (Hines and Garvey 2006), and sequential probability ratio test (SPRT) (Wald 1945). EULM fault detection adapts simple thresholding for use in the nuclear power industry by monitoring the uncertainty bounds about a sensed value (or state estimation residual) and alarming when the uncertainty bounds exceed some threshold. This approach offers an additional level of conservatism to the fault detection, indicating when the monitored parameter is no longer within the error thresholds to some specified confidence level. SPRT considers a sequence of residuals and determines if they are more likely from the distribution that represents normal behavior or that of a faulted distribution, which may have a shifted mean value or altered standard deviation from the nominal distribution. While these fault detection methods are used to determine if a fault is present in the system, fault isolation involves determining where the fault has occurred. In the context of sensor calibration monitoring, this involves identifying which sensor has 
degraded in order to target sensor recalibration efforts. Fault isolation, also referred to as diagnostics, is typically performed with pattern recognition methods, rule-based methods, or expert systems.

One important research area for all OLM models is understanding and quantification of uncertainty. In response to specific proposals to apply OLM in operating nuclear power plants (EPRI 1998), aspects of uncertainty quantification were part of the requirements identified by the regulator as needing resolution from utilities (EPRI 2000a; Hines and Seibert 2006a).

\subsection{Current Approaches to Uncertainty for OLM}

In OLM approaches to calibration assessment, the expected, nominal sensor measurement is calculated from a model of the system's normal behavior. As in any modeling paradigm, these predictions have some level of associated uncertainty. Understanding and quantifying this uncertainty is a key need in developing an OLM system for sensor performance monitoring. In order to apply OLM in NPPs, the sources of uncertainty must be quantitatively bounded and accounted for in the calibration acceptance criteria or in the trip setpoint and uncertainty calculations (EPRI 2008).

Hashemian (1995a) states that, if the process estimate uncertainty is stationary in time and constant across the measurement range, the estimate uncertainty does not affect the ability to detect sensor drift. However, these uncertainties are important for determining the level of deviation that is acceptable (calibration acceptance criteria). The EULM drift detection method, which was proposed for sensor drift monitoring, monitors the uncertainty band about the parameter residual to determine if a sensor is no longer in its acceptable calibration tolerance.

A review of estimating modeling uncertainty for a variety of empirical modeling methods is given in Rasmussen (2003). Rasmussen uses prediction intervals to quantify and bound the parameter estimate uncertainty. However, many sensors contain a significant amount of measurement noise that causes the prediction interval uncertainty estimate to exceed the drift allowance even when no drift is present (Seibert et al. 2006). To alleviate this burden, application of confidence intervals (with certain assumptions) to the denoised (filtered) residuals to detect sensor drift is proposed. However, redundant sensor models typically do not meet some of the assumptions (Hines et al. 2008b), and the appropriate uncertainty interval depends on the modeling method architecture (i.e., redundant or non-redundant sensor models).

Regardless of the approach, the OLM uncertainty is typically characterized by the bias/variance decomposition of the model error (Tamhane and Dunlop 2000). Given a model with prediction variance $\operatorname{Var}(\hat{x})$ and Bias representing the systematic error of the model (Hines et al. 2008a), the total uncertainty is a combination of the prediction variance, the model bias, and the irreducible error (generally considered noise):

$$
U(\hat{x})=\operatorname{Var}(\hat{x})+\text { Bias }^{2}+\sigma_{\text {noise }}^{2}
$$

When it is appropriate to the modeling architecture, the noise can be removed from the uncertainty by denoising the model prediction residuals. The prediction variance portion of uncertainty can be calculated through analytic or Monte Carlo techniques (Hines and Rasmussen 2005). 
Analytic uncertainty is estimated by closed-form equations derived from the model's mathematical architecture. Such equations have been derived for several models, including the multivariate state estimation technique (MSET), auto-associative kernel regression (AAKR), linear regression, etc. (Hines et al. 2008a). Monte Carlo uncertainty estimation applies a resampling technique to sample the training data multiple times and construct a bank of models. The variation between the predictions of all the models is used as the prediction variance portion of the total uncertainty estimation. Monte Carlo methods measure the uncertainty across a population of possible models, while analytic methods estimate the uncertainty of the current model; as such, the Monte Carlo estimate of prediction variance tends to be slightly larger. Both techniques have been shown to be conservative (Rasmussen 2003).

The total uncertainty plays a key role in determining sensor calibration acceptance criteria and trip setpoints. In early work, the channel statistical accuracy (CSA) was used to determine the trip setpoints for the plant and the acceptance criteria for OLM. The CSA is an estimate of the total channel uncertainty, and includes process measurement accuracy, calibration accuracy, an allowance for sensor drift, pressure and temperature effects, etc. The OLM acceptance criteria is then the CSA less the uncertainty of the parameter prediction (Hashemian 1995a). More recently Hines and Seibert (2006a) defined the acceptance criteria, called the allowable deviation value for online monitoring (ADVOLM), as:

$$
A D V O L M=\sqrt{\left(S D^{*}+S M T E+S C A\right)^{2}-O L M P E_{U N C}^{2}-S P M A^{2}},
$$

where $S D^{*}$ is the potential sensor drift during the surveillance interval, SMTE is the sensor measurement and test equipment accuracy, $S C A$ is the sensor calibration accuracy, OLMPE $E_{U N C}$ is the uncertainty in the parameter prediction, and SPMA is the single point monitoring allowance. In this formulation, $\left(S D^{*}+S M T E+S C A\right)$ is similar to the CSA above. SPMA arises from the expected steady-state operation of the plant during OLM, and is used to account for potential variation in OLM assessment across the range of the sensor (as typically, the sensor calibration is being evaluated in OLM algorithms only at the operating conditions, while it is evaluated across the full sensor range in traditional calibration assessment). 


\subsection{A Data-Driven Approach to Uncertainty Quantification}

While online monitoring for sensor calibration monitoring and interval extension has been an active area of research for the past 25 years, several gaps remain to bring this technology to industry. A key gap is the need for better UQ approaches. Instrument trip setpoints and acceptance criteria for calibration both need to account for the uncertainty inherent in OLM, providing some prescribed degree of confidence that a sensor is operating within its calibration specification.

Several sources of uncertainty will contribute to the overall result. Methods for quantifying the total uncertainty are needed to address the remaining gaps in this area. Data-driven approaches are attractive in this regard as they can potentially determine changes in the confidence bounds as conditions change in the plant.

\subsection{Specific Sources of Uncertainty in OLM}

Through review of the relevant literature, several sources of uncertainty important to online sensor calibration monitoring and signal validation have been identified (Hashemian 1995b; EPRI 2000b; Hines and Seibert 2006b). These uncertainty sources can be roughly categorized as (1) process noise, (2) measurement uncertainty, (3) electronic noise, and (4) modeling uncertainty. The four sources of uncertainty are briefly described below.

Process Noise - Process noise is the result of normal fluctuation of the physical process parameters (e.g., temperature, flow, pressure) about the true process value. This can be from flow characteristics, incomplete mixing, or local variations. Often, process noise is common (or approximately common) to co-located, redundant sensors.

Measurement Uncertainty - Measurement uncertainty is due to a number of factors, including sensor accuracy, calibration accuracy (e.g., calibration offset, error in conversion from sensor units to engineering units), environmental effects (because of temperature, vibration, pressure, etc.). These uncertainty sources apply to both the sensor and to the rack/isolator.

Electronic Noise - Transmission of measurements down the instrumentation line can induce additional noise, along with the analog-to-digital conversion at the computer input. Transmission line noise may change as instrumentation cables age.

OLM Model Prediction Uncertainty - The results of OLM models have associated uncertainty. The prediction uncertainty stems from both input uncertainty (related to the process noise, measurement uncertainty, and electronic noise outlined above) and modeling error (arising from model selection, model training, input selection, etc.). Depending on the modeling algorithm, closed-form (analytic) uncertainty estimates may be derived; for example, uncertainty estimates for linear regression models are well understood. In the absence of analytic uncertainty formulas, Monte Carlo-based estimates can be made (indeed, these bootstrap estimates can be made for any model type, although they are more computationally intensive).

OLM calibration assessment routines typically evaluate model residuals (the difference between sensor measurements and predicted values) to detect and identify sensor faults. The total residual 
uncertainty should account for uncertainty in measurements and in model predictions. To understand and evaluate the propagation of uncertainty from measurements through predictions and residuals, both mathematical simulations and experiments are proposed to develop a method to characterize OLM uncertainty.

\subsection{General Principles of Uncertainty Quantification}

Uncertainty quantification (UQ) is the science of quantitative characterization and reduction of uncertainties in applications. It tries to determine how likely certain outcomes are if some aspects of the system are not exactly known.

Uncertainty can enter mathematical models and experimental measurements in various contexts. One way to categorize the sources of uncertainty is to consider (Kennedy and O'Hagan 2001):

- Parameter uncertainty, which comes from the model parameters that are inputs to the computer model (mathematical model) but whose exact values are unknown to experimentalists and cannot be controlled in physical experiments. Examples are the local free-fall acceleration in a falling object experiment, and various material properties in a finite element analysis for mechanical engineering.

- Structural uncertainty, aka model inadequacy, model bias, or model discrepancy, which comes from the lack of knowledge of the underlying true physics. It depends on how accurately a mathematical model describes the true system for a real-life situation, considering the fact that models are almost always only approximations to reality. One example is when modeling the process of a falling object using the free-fall model; the model itself is inaccurate because there always exists air friction. In this case, even if there is no unknown parameter in the model, a discrepancy is still expected between the model and true physics.

- Algorithmic uncertainty, aka numerical uncertainty, which comes from numerical errors and numerical approximations per implementation of the computer model. Most models are too complicated to solve exactly. For example, the finite element method or finite difference method may be used to approximate the solution of a partial differential equation, which, however, introduces numerical errors. Other examples are numerical integration and infinite sum truncation that are necessary approximations in numerical implementation.

- Parametric variability, which comes from the variability of input variables of the model. For example, the dimensions of a work piece in a process of manufacture may not be exactly as designed and instructed, which would cause variability in its performance.

- Experimental uncertainty, aka observation error, which comes from the variability of experimental measurements. The experimental uncertainty is inevitable and can be noticed by repeating a measurement for many times using exactly the same settings for all inputs/variables.

- Interpolation uncertainty, which comes from a lack of available data collected from computer model simulations and/or experimental measurements. For other input settings that do not have simulation data or experimental measurements, one must interpolate or extrapolate in order to predict the corresponding responses.

Another way of categorization is to classify uncertainty into two categories (Matthies 2007; Kiureghiana and Ditlevsen 2009): 
- Aleatoric uncertainty, aka statistical uncertainty, which is unknowns that differ each time we run the same experiment. For an example of simulating the take-off of an airplane, even if we could exactly control the wind speeds along the run way, if we let 10 planes of the same make start, their trajectories would still differ because of fabrication differences. Similarly, if all we knew is that the average wind speed is the same, letting the same plane start 10 times would still yield different trajectories because we do not know the exact wind speed at every point of the runway, only its average. Aleatoric uncertainties are therefore something an experimenter cannot do anything about: they exist, and they cannot be suppressed by more accurate measurements.

- Epistemic uncertainty, aka systematic uncertainty, which is from things we could in principle know but do not in practice. This may be because we have not measured a quantity sufficiently accurately, or because our model neglects certain effects, or because particular data are deliberately hidden.

In real-life applications, both kinds of uncertainties are often present. Uncertainty quantification intends to work toward reducing epistemic uncertainties to aleatoric uncertainties. The quantification for the aleatoric uncertainties is relatively straightforward to perform. Techniques such as the Monte Carlo method are frequently used. A probability distribution can be represented by its moments (in the Gaussian case, the mean and covariance suffice), or more recently, by techniques such as KarhunenLoève and polynomial chaos expansions. To evaluate epistemic uncertainties, the efforts are made to gain better knowledge of the system, process, or mechanism. Methods such as fuzzy logic or evidence theory (Dempster-Shafer theory - a generalization of the Bayesian theory of subjective probability) are used.

Parameter estimate uncertainty could come from many contributing sources. For empirical modeling techniques, inaccuracies in the training data and inherent limitations of the model contribute to the total uncertainty. For parity spaced-based methods, the user-defined inputs, the number of redundant channels, and the noise level and amount of drift present in each channel contribute to the uncertainty of the parameter estimate.

\subsection{Uncertainty Analysis Assumptions}

There are also several assumptions made in the application of the various uncertainty analysis algorithms in this study. These include:

- Monte Carlo methods make use of an estimate of the "true value" of the sensor.

- Measurement data is assumed to be stationary.

- The analytical techniques used to date assume that the variance portion of uncertainty is much larger than the bias portion.

These assumptions and their effect on predictive uncertainty must be investigated and quantified. Future research will examine and attempt to quantify the overall effect of this assumption. 


\subsection{Uncertainty Quantification Methods for Uncertainty Estimation in Online Sensor Calibration Monitoring}

\subsubsection{Polynomial Chaos Methods}

Polynomial chaos (PC), also called "Wiener Chaos expansion" (Wiener 1938), is a non-samplingbased method to determine evolution of uncertainty in a dynamical system, when there is probabilistic uncertainty in the system parameters.

PC was first introduced by Wiener where Hermite polynomials were used to model stochastic processes with Gaussian random variables. It can be thought of as an extension of Volterra's theory of nonlinear functionals for stochastic systems. Such an expansion converges in the $\mathrm{L}_{2}$ sense for any arbitrary stochastic process with finite second moment (Ghanem and Spanos 1991). This applies to most physical systems. Xiu (2010) generalized the result of Cameron-Martin to various continuous and discrete distributions using orthogonal polynomials from the so-called Askey-scheme and demonstrated $\mathrm{L}_{2}$ convergence in the corresponding Hilbert functional space. This is popularly known as the generalized polynomial chaos (gPC) framework. The gPC framework has been applied to applications including stochastic fluid dynamics, stochastic finite elements, solid mechanics, nonlinear estimation, and probabilistic robust control. It has been demonstrated that gPC-based methods are computationally superior to Monte Carlo-based methods in a number of applications. However, the method has a notable limitation. For large numbers of random variables, polynomial chaos becomes very computationally expensive and Monte Carlo methods are typically more feasible.

\subsubsection{Gaussian Process Model}

In probability theory and statistics, a Gaussian process is a stochastic process whose realizations consist of random values associated with every point in a range of times (or of space) such that each such random variable has a normal distribution. Moreover, every finite collection of those random variables has a multivariate normal distribution.

A Gaussian process can be used as a prior probability distribution over functions in Bayesian inference (Rasmussen and Williams 2006; Liu et al. 2010). Given any set of N points in the desired domain of your functions, take a multivariate Gaussian whose covariance matrix parameter is the Gram matrix of your $\mathrm{N}$ points with some desired kernel, and sample from that Gaussian.

Inference of continuous values with a Gaussian process prior is known as Gaussian process regression, or kriging; extending Gaussian process regression to multiple target variables is known as cokriging (Stein 1999). As such, Gaussian processes are useful as a powerful non-linear interpolation tool. Additionally, Gaussian process regression can be extended to address learning tasks both in a supervised (e.g., probabilistic classification (Rasmussen and Williams 2006)) and an unsupervised (e.g., manifold learning) learning framework.

Physical systems usually have responses that consist of a set of distinct outputs (e.g., velocity, temperature, and pressure) that evolve also in space and time and depend on many unknown input parameters (e.g., physical constants, initial conditions, etc.). Furthermore, essential engineering procedures such as uncertainty quantification, inverse problems, or design are notoriously difficult to 
carry out mostly because of the limited simulations available. The aim of this work is to introduce a fully Bayesian approach for treating these problems that accounts for the uncertainty induced by the finite number of observations.

Our model is built on a multi-dimensional Gaussian process that explicitly treats correlations between distinct output variables as well as space and/or time. In this way, we obtain error bars for the statistics of interest that account for the finite number of observations. The novelty of this work is to introduce a Gaussian process model into the power plant monitoring. We use this model to build boundaries of the steady-state process to obtain error bars for the statistics of interest that account for the finite number of observations. We can also detect deviations from the steady-state process statistics (mean, variance, correlation parameters, etc.) for the variables of interest.

Let us consider a physical problem with input domain $X_{\xi} \subset R^{k_{\xi}}$, spatial domain $X_{s} \subset R^{k_{s}}$, and temporal domain, which is expressed as an interval XT=[0,T], where $k_{\xi}, k_{s}$ are the dimensions of the input and spatial domain. The input domain $X_{\xi}$ usually represents a bounded domain and can thus be considered a compact subset of $R^{k_{\xi}}$ while the spatial domain $X_{s}$ and time domain $T$ can be given intervals on $R^{k_{s}} \times R^{+}$.

In the computer simulations, we usually fix the spatial and temporal domain and sample the input domain. This gives us computational flexibility because we can represent the domain as a tensor product of the input, spatial, and temporal domain. For an input parameter $\xi \in X_{\xi}$, the computer simulation returns the (multi-output) response on a given (a priori known) set of $n_{s}$ spatial points $X_{s}=\left(s_{1}, \ldots, s_{n_{s}}\right)^{T} \in R^{n_{s} \times k_{s}}$, where $k_{s}=1,2$, or 3 is the number of spatial dimensions, at each one of the $n_{t}$ timesteps $X_{t}=\left(t_{1}, \ldots, t_{n_{t}}\right) \in R^{n_{t} \times 1}$. That is, a single choice of the input domain $\xi$ generates a total of $n_{s} \times n_{t}$ training samples. Therefore, the response is a matrix in $R^{\left(n_{s} n_{t}\right) \times q}$, where $q$ is the number of the output variables of the computer simulation.

For modeling reasons, we will represent the problem as a $q$ multivariate response $\left(\xi_{i}, s_{i}, t_{i}\right) \in R^{q}$ given input, spatial and time point $i=\left(\xi_{i}, s_{i}, t_{i}\right)$. We denote the multiple observed output vector as $Y=\left(\beta\left(x_{1}\right)^{T}, \ldots, \beta\left(x_{N}\right)^{T}\right)^{T}$ and $X=\left(x_{i}^{T}, \ldots, x_{i}^{T}\right)$ as its corresponding input, spatial, and time vector. For simplification purposes, we call the input domain, spatial domain, and temporal domain as input, space, and time, respectively. Throughout this report we will collectively denote input of $\beta(\cdot)$ by $x=(\xi, s, t)$ and the space domain by $X \equiv X_{\xi} \times X_{s} \times[0, T]$. Finally, we denote $N=n_{\xi} \times n_{s} \times n_{t}$ as the total sample size of the new setting.

Gaussian Process (GP) is a tool that has been successful building surrogate models for the computer simulations. The challenge in GP is to model the mean and the variance. A linear regression model for the mean is usually a good choice for continuous fields. The covariance function is harder to model and usually depends on the computational complexity and the form of $\beta(\cdot)$. In this report, we use a combination of linear model of coregionalizations and the separable model. The Bayesian formulation 
assumes a GP prior distribution for the function $\beta(\cdot)$, conditional on various hyperparameters. This prior distribution is updated using a preliminary training sample on input domain $X_{\xi} \times X_{s} \times[0, T]$. The goal of this work is to interpolate within the Bayesian framework a multivariate function $(\cdot): R^{\xi} \times R^{k_{s}} \times R^{+} \rightarrow R^{q}$ based on some observations.

The classical multivariate Gaussian process can be written as:

$$
\eta(x)=\mu(x)+w(x)+\varepsilon(x)
$$

where $\mu(x)$ is the mean which is usually modeled as a linear regression, $w(x)$ is the spatial correlation, and $\varepsilon(x)$ denotes the nugget error. Modeling the cross-covariance function of $w$ is one of the most important questions we have to answer. In what follows, we give different strategies to model the cross-covariance of $w$.

\subsubsection{Estimates of Noise on Sensors and UQ Methods for De-noising}

The current approach to quantifying uncertainty in nuclear instrumentation relies on estimates of noise and environmental effects on sensors, typically provided by the manufacturer (Hashemian 1995a). Combined instrument channel uncertainty is then calculated according to ISA-RP67.04.02-2010 (ISA 2010). This approach provides a conservative estimate of the channel uncertainty, which then affects the plant trip setpoints and acceptable operating envelopes. OLM introduces additional uncertainties, such as model prediction uncertainty (Hines and Seibert 2006a), which must also be accounted for in assessing the accuracy of sensors and instrumentation. Methods of UQ based on the measured data are being investigated to alleviate some of the conservatism inherent in the current approach.

One of the major issues is the technique used to provide what Argonne National Laboratory (ANL) calls "Perfect Signal Reconstruction.” These "perfect” or noise-free signals are needed to estimate the bias term in the uncertainty analysis, as well as to quantify the noise, and its distribution, present in each sensor input. The technique, termed the Stochastic Parameter Simulation System (SPSS), is a waveletbased technique originally developed by Miron (2001) to whiten residuals for input to the Sequential Probability Ratio Test (SPRT), which was used in the past for drift detection. The SPRT is developed under the assumptions of white residuals, meaning that each residual is uncorrelated with itself over time. The SPSS method was developed as an alternative to the Reactor Parameter Signal Simulator (RPSS) developed at ANL (Gross and Hoyer 1993) for SPRT residual whitening. The SPSS was also used to estimate noise-free signals so that noise distributions could be quantified and the Latin Hypercube Sampling technique could be applied.

The ICA algorithm, which has been applied to sensor calibration monitoring (Ding 2004), could be used as an alternative to the wavelet-based approach. It is assumed that because the sensors in OLM are all redundant, the ICA technique will perform as well as, or better than, the wavelet de-noising technique in estimating the true process measurement. ICA is only applicable to stationary signals. However, with single-point monitoring in effect, it is expected to be a valid technique. Miron's SPSS program is used to analyze steady-state plant signals. It decomposes the signal into its deterministic and stochastic components, and then reconstructs a new, simulated signal that possesses the same statistical noise characteristics as the actual signal for use in the Monte Carlo-based uncertainty analysis. It is also used as 
a filtering device. For filtering, it isolates the principal serially correlated, deterministic components from the analyzed signal so that the remaining stochastic signal can be analyzed with signal validation tools. The wavelet de-noising function decomposes the signal into its low-frequency (approximation) and highfrequency (detail) components.

\subsubsection{Summary}

The proposed UQ methods can statistically determine the uncertainty of a model at a single operating point. Comparing to the classic Monte Carlo methods, the proposed UQ methods can greatly improve the predictive capability and the accuracy of predicting uncertainty with the same number of samples. We are evaluating the proposed UQ methods using data from simulations and experimental flow loops. The results of these analyses will be compared to the uncertainty results of the traditional method to evaluate any potential gains. 



\subsection{Preliminary Results}

This section describes preliminary results of the data-driven uncertainty quantification approach. Two approaches were taken for generating the data sets needed for this evaluation. Experimental data was acquired using an instrumented flow loop, with varying test conditions. In addition, a simulation model of a flow-loop was generated. The simulation model serves two purposes. First, data may be generated from the simulation model that represent conditions the experimental flow loop may not be able to achieve. Second, the use of a model can provide insights into physical relationships between measurements from sensors at different locations. These insights can be used to derive new algorithms for OLM, as well as develop methods for generating virtual sensors. Although analysis of data generated from the simulation models is continuing, the models themselves are included in this section for completeness.

\subsection{Experimental Data for Evaluation of UQ Methods}

\subsubsection{Flow Loop Configuration}

An instrumented flow loop was used to produce the data for analysis. The 17 sensors in the loop consist of pressure and differential pressure transmitters to measure pressures and flows, and a combination of thermocouples and RTDs to measure temperatures. A schematic of the loop along with a table of the instruments used is shown in Figure 4.1. Water is pumped through the loop by a 7.5-HP centrifugal pump, shown at the bottom left of the figure. The water flow direction is clockwise relative to the figure. The primary loop is constructed of 4-inch Schedule 40 PVC pipe, the heat exchanger lines are 1.5-inch Schedule 40 PVC pipe, and the sensing lines are 0.25-inch copper tubing.

Because this is a closed loop, heat generated by the pump must be removed to prevent temperature runaway. To accomplish this, water is diverted to an air-cooled heat exchanger via a diverting valve that is controlled by a temperature controller. The temperature controller senses the water temperature via an RTD at location \#10 on the diagram, and positions the diverting valve based on cooling demand. The cooled water leaves the heat exchanger and mixes back with the process water near the pump inlet, between locations 14 and 15 on the diagram. The heat exchanger is cooled by ambient air via a 2-HP forced-air fan. Because the heat exchanger is cooled by ambient air, the minimum operating temperature of the water in the loop is limited to about $10-15^{\circ} \mathrm{F}$ above the local outdoor ambient temperature.

Similar to temperature, pressure is controlled by a pressure controller. The controller senses static gauge pressure at location 14 on the diagram, and its outputs are connected to two normally closed solenoid valves. A solenoid valve located near the pump inlet raises system pressure by letting water enter the system from the building water supply, and a second solenoid valve located at the highest point

in the system releases pressure via a vent line to the atmosphere. Note that the maximum system pressure is limited to both the building supply water pressure and the maximum pressure rating of the PVC piping, which is about 80 PSIG. 
I\&C026-10

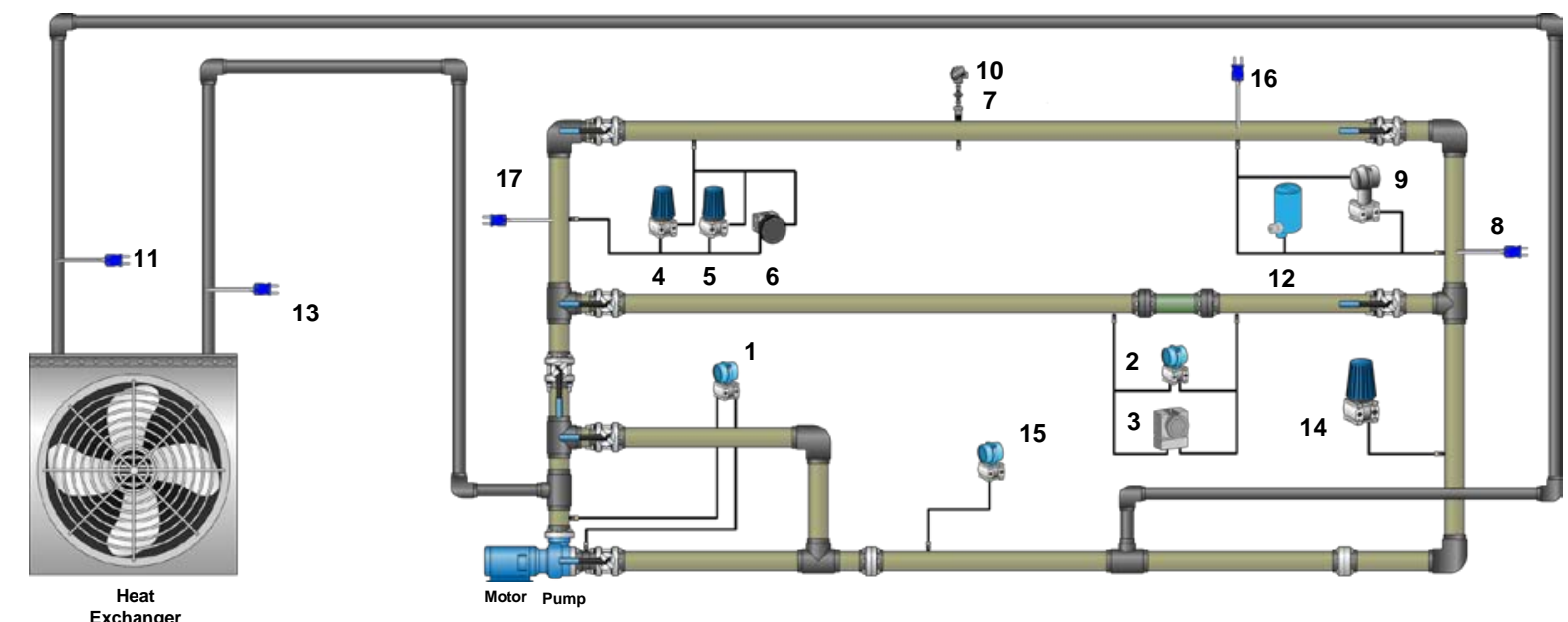

\begin{tabular}{|c|c|c|c|c|c|c|c|}
\hline ITEM & ID & SENSOR TYPE & MANUFACTURER & ITEM & ID & SENSOR TYPE & MANUFACTURER \\
\hline 1 & FT-4-1 & DIFFERENTIAL PRESSURE & ROSEMOUNT & 10 & CTRL-TEMP & RTD (SMART) & ROSEMOUNT \\
\hline 2 & FT-3-1 & DIFFERENTIAL PRESSURE (SMART) & ROSEMOUNT & 11 & TC-HX-OUT & THERMOCOUPLE TYPE-J & OMEGA \\
\hline 3 & FT-3-2 & DIFFERENTIAL PRESSURE & BARTON & 12 & FT-2-3 & DIFFERENTIAL PRESSURE & HONEYWELL \\
\hline 4 & FT-1-1 & DIFFERENTIAL PRESSURE & FOXBORO & 13 & TC-HX-IN & THERMOCOUPLE TYPE-J & OMEGA \\
\hline 5 & FT-1-2 & DIFFERENTIAL PRESSURE & FOXBORO & 14 & CTRL-PSR & GAUGE PRESSURE & FOXBORO \\
\hline 6 & FT-1-4 & DIFFERENTIAL PRESSURE (SMART) & BARTON & 15 & PT-2 & GAUGE PRESSURE & ROSEMOUNT \\
\hline 7 & TE-1-2 & RTD (SMART) & ROSEMOUNT & 16 & TC-LOOP-FAR & THERMOCOUPLE TYPE-E & OMEGA \\
\hline 8 & TC-2-1 & THERMOCOUPLE TYPE-J (SMART) & ROSEMOUNT & 17 & TC-PUMP-OUT & THERMOCOUPLE TYPE-K & OMEGA \\
\hline 9 & FT-2-1 & DIFFERENTIAL PRESSURE & SCHLUMBERGER & & & & \\
\hline
\end{tabular}

Figure 4.1. Flow Loop Piping and Instrumentation Diagram

\subsubsection{Data Collection Method}

Data were collected from the sensors via Analysis and Measurement Services' OLM-32 data acquisition system and its accompanying software. A picture of the OLM-32 system is shown in Figure 4.2. The OLM-32 hardware is capable of sampling data with 24-bit resolution at a maximum rate of $50 \mathrm{kHz}$, although data for this project were collected at 200, 20, and $10 \mathrm{~Hz}$ (depending on the test).

Each sensor output was routed through a transmitter before reaching the data acquisition system. The transmitters converted the raw sensor output to a 1-5 V signal. 


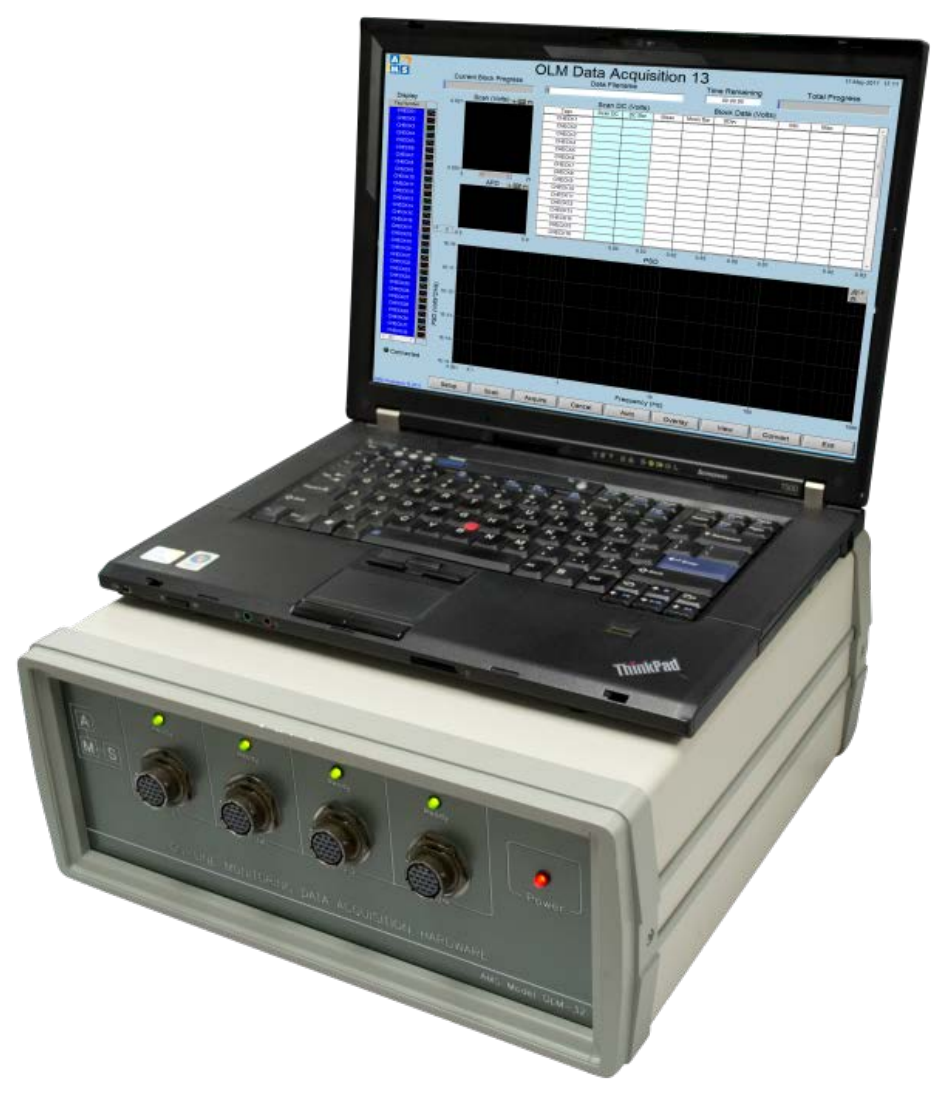

Figure 4.2. OLM-32 Data Acquisition System

\subsubsection{Test Conditions}

Testing was generally conducted at steady-state conditions. As previously mentioned, the steadystate temperature of the loop depends on the ambient temperature (because of the ambient air-cooled heat exchanger). Testing was conducted during the months of July and August where the daytime temperatures averaged around $85^{\circ} \mathrm{F}$. This limited the minimum operating temperature of the loop to about $95^{\circ} \mathrm{F}$; therefore, $100^{\circ} \mathrm{F}$ was used as the steady-state condition for most of the tests.

As previously mentioned, pressure was maintained by a pressure controller located on the operator control panel. Because building water pressure was used as the pressure source, and to prevent excess stress to the PVC piping, the pressure was limited to about 80 PSIG. The steady-state pressure setpoint was 55 PSI.

\subsubsection{OLM Algorithm}

Empirical models of the test loop data were created using the auto-associative kernel regression technique. AAKR is a memory-based empirical modeling technique that attempts to predict noise-free estimates of the given input signals where the inputs and outputs are of the same dimensions. Figure 4.3 shows a block diagram of the basic AAKR model with three inputs and three outputs. 


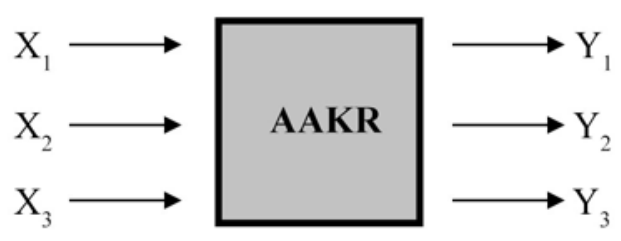

Figure 4.3. AAKR Model with Inputs $X$ and Outputs $Y$

AAKR stores its fault-free training data in an NxP memory matrix $\mathbf{M}$, which is made up of $\mathrm{N}$ observations of $\mathrm{P}$ process variables:

$$
M=\left[\begin{array}{cccc}
M_{1,1} & M_{1,2} & \ldots & M_{1, P} \\
M_{2,1} & M_{2,2} & \ldots & M_{2, P} \\
\ldots & \ldots & \ldots & \ldots \\
M_{N, 1} & M_{N, 2} & \ldots & M_{N, P}
\end{array}\right]
$$

For each input vector $\mathrm{x}_{\mathrm{i}}=\left[\begin{array}{llll}\mathrm{x}_{1} & \mathrm{x}_{2} & \ldots & \mathrm{x}_{\mathrm{P}}\end{array}\right]$ presented to the AAKR model, the distance between $\mathrm{x}_{\mathrm{i}}$ and each vector in the memory matrix $\mathrm{M}$ is calculated according to a distance function, such as the Euclidean distance given by:

$$
d_{i}\left(\mathbf{M}_{i}, \mathbf{x}\right)=\sqrt{\left(M_{i, 1}-x_{1}\right)^{2}+\ldots+\left(M_{i, p}-x_{p}\right)^{2}}
$$

For each input vector $x_{i}$, the calculation in Eq. (4.2) results in a vector $d_{i}=\left[\begin{array}{lllll}d_{1} & d_{2} & \ldots & d_{N}\end{array}\right]$ made up of the distances between the input vector $\mathrm{x}_{\mathrm{i}}$ and all of the vectors in the memory matrix $\mathrm{M}$. Next, these distances are used to calculate a weighting vector $\mathrm{w}_{\mathrm{i}}$ according to a similarity function, typically given by the Gaussian kernel function:

$$
w_{i}=K_{b}\left(d_{i}\right)=e^{-d^{2} / b^{2}},
$$

where $b$ is the kernel bandwidth. This effectively gives a higher weight to memory matrix vectors that are closer to the input vector $\mathrm{x}_{\mathrm{i}}$. Finally, these weights are used to produce the output prediction, $\mathrm{y}_{\mathrm{i}}$ according to:

$$
\mathbf{y}_{i}=\frac{\sum_{i=1}^{N} w_{i} M_{i}}{\sum_{i=1}^{N} w_{i}},
$$

where the output prediction $\mathrm{y}_{\mathrm{i}}$ is a weighted average of memory vectors in $\mathrm{M}$. The difference between each model prediction, $\mathbf{y}_{\mathrm{i}}$ and the corresponding input vector $\mathrm{x}_{\mathrm{i}}$ form a residual vector, $\mathbf{r}_{\mathrm{i}}$. Each individual element of the residual vectors represents the difference between the model prediction and the actual measured value, and can be compared to a threshold value to determine if the measured values are outside the bounds of normal operation as characterized by the training data. 
For the test loop data, AAKR models consisting of data from all 17 sensors of the test loop were created using fault-free data. The models were then presented with measured values under various faultfree and fault conditions for each test that was performed. The residuals for each test were then calculated and compared to threshold values. Threshold values of $\pm 1 \%$ of the calibrated range of each sensor were used to determine if the measured values deviated significantly from normal operation.

\subsection{Uncertainty Analysis and Quantification}

Data from the instrumented flow loop were used for initial assessment of the proposed UQ methodology. The flow loop operated at steady state for each setpoint for at least five minutes. The steady-state data, taken from the no-fault cases, was used for this initial assessment. The data was divided into a training set and a verification set, where the training set data were at several temperature setpoints varying from $90^{\circ} \mathrm{F}$ to $110^{\circ} \mathrm{F}$ and various pressure setpoints, with the sample rate set to $20 \mathrm{~Hz}$. We use these data to obtain error bars for the statistics of interest that account for the different values of initial conditions.

Conditioned on some hyperparameters that control the mean and the variance, we assume that our data follow a multivariate normal distribution. The Bayesian formulation assumes a Gaussian prior distribution for the function $\beta(\cdot)$ conditional on the hyperparameters. This prior distribution is updated using preliminary training data, as a function of the input domain (temperature and/or pressure setpoints) and time. The overall goal is to give an analytical representation of the response surface of the multivariate function $\beta(\cdot)$ for arbitrary input values and time based on some observations.

Because we can exactly specify the prediction (posterior) distribution of $\beta(\cdot)$ at an arbitrary input $x$ at time $t$ given the data and the hyperparameters, we can calculate the prediction distribution of $\beta(\cdot)$ at an arbitrary input $x$ at time $t$ given only the data by Bayesian model averaging (Hoeting et al. 1999).

An example of the type of results that may be obtained from this process is shown in Figure 4.4. The data shown in this figure presents the prediction estimates of each of the sensor responses as a function of temperature (assuming that the input variable is temperature only). Each sensor prediction shows the predicted value (the central curve in each plot) and the prediction error bounds (lower and upper curves). Note that the data presents a snapshot in time; that is, the predicted values shown are at a single instant in time. Similar predictions may be made for other time instances.

Clearly, the ability to rapidly compute such error bounds provides the capability for quantifying the uncertainty from the data itself, assuming the model postulated (Gaussian Process). Clearly, for OLM, sensor drift or faults will have to result in measurements that exceed these bounds, which are computed from no-fault data. The assessment of data from faulted sensors in this framework is ongoing. 

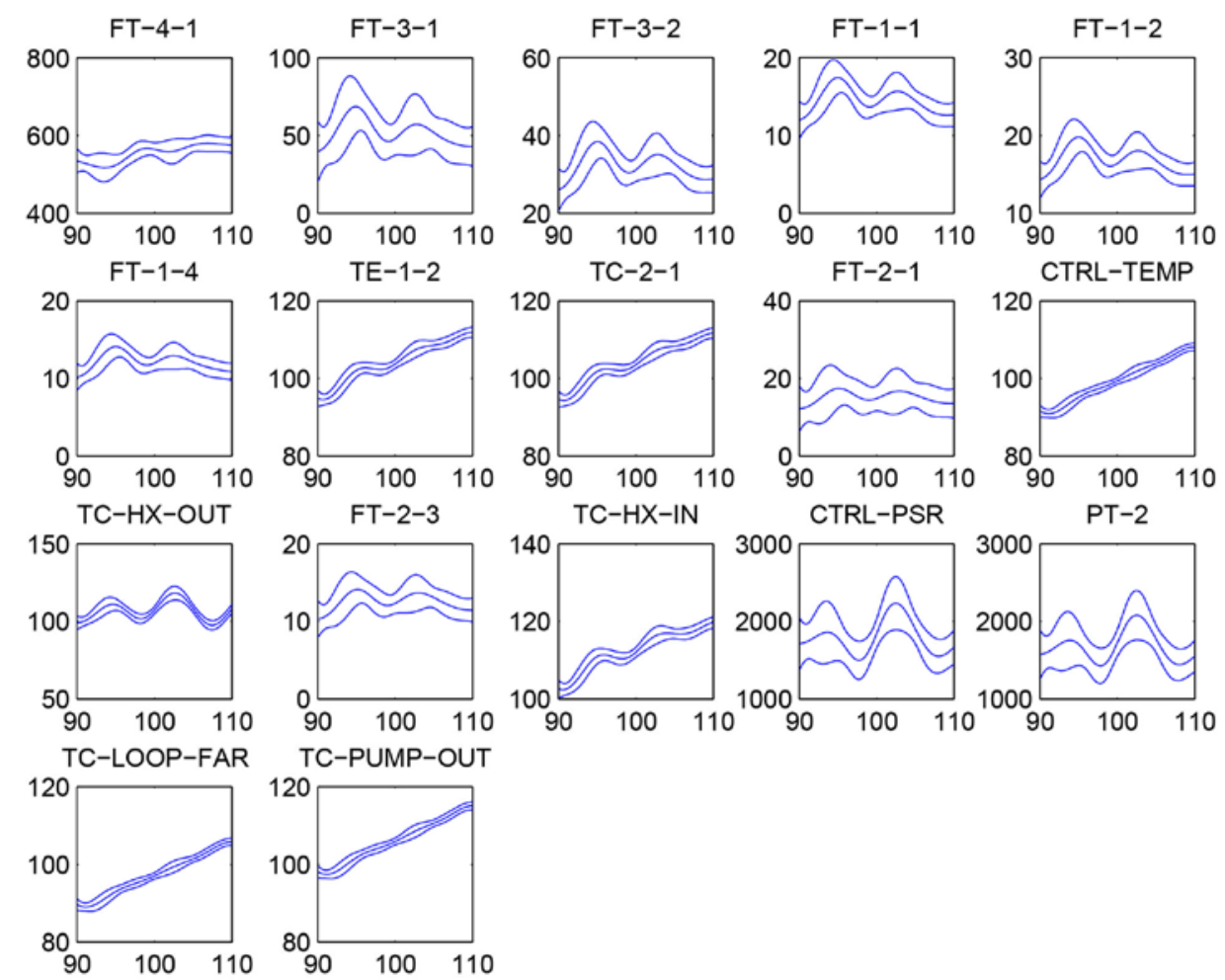

Figure 4.4. Snapshot of Predictions for All Sensor Data, Assuming a Single Input Variable (temperature). The horizontal axis of each plot shows temperature in ${ }^{\circ} \mathrm{F}$. The vertical axes show, depending on the sensor type, either temperature $\left({ }^{\circ} \mathrm{F}\right)$ or differential pressure (inches $\mathrm{H}_{2} \mathrm{O}$ ).

The ability to extend this approach to all OLM models is not clear. However, the proposed approach may be seen as a generalization of AAKR models for OLM that use Gaussian kernels in a Basis expansion process (Section 4.1.4), as the proposed GP model can address covariance and correlations between the different sensor measurements much more efficiently, using the same basic framework of Gaussian processes. As a result, it is expected that the data-driven approach proposed here can be readily extended to AAKR OLM models, and potentially to a larger subset of OLM models as well, although this needs further verification.

To further assess the approach, we performed an analysis where two input variables (temperature and differential pressure) were assumed. Figure 4.5 shows the results of the prediction at time $t=4990$ seconds. The left column presents the observed data as a function of temperature (horizontal axis) and differential pressure (vertical axis), with the values of the sensor data color-coded using the color-bar on the right. The response surface (prediction) for the corresponding sensor is presented in the right column. The data is presented for 9 of the 17 sensors used in the instrumented flow loop. As with the 1-D response surfaces, only steady-state data from the no-fault conditions are used in the generation of the response surfaces. Note that the prediction accuracy appears to be reasonable, although confidence bounds are not presented in this particular example. Additional analysis is ongoing to determine the impact of using a larger data set and approaches to visualizing the error bars. 


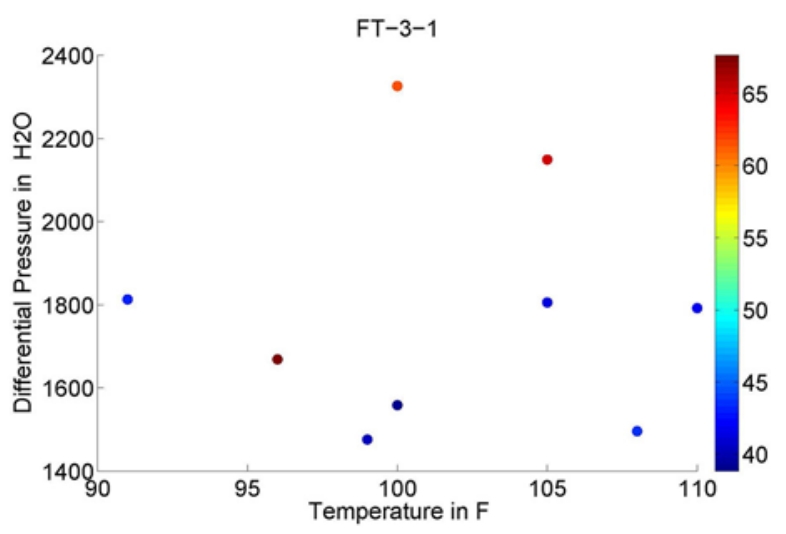

(a)

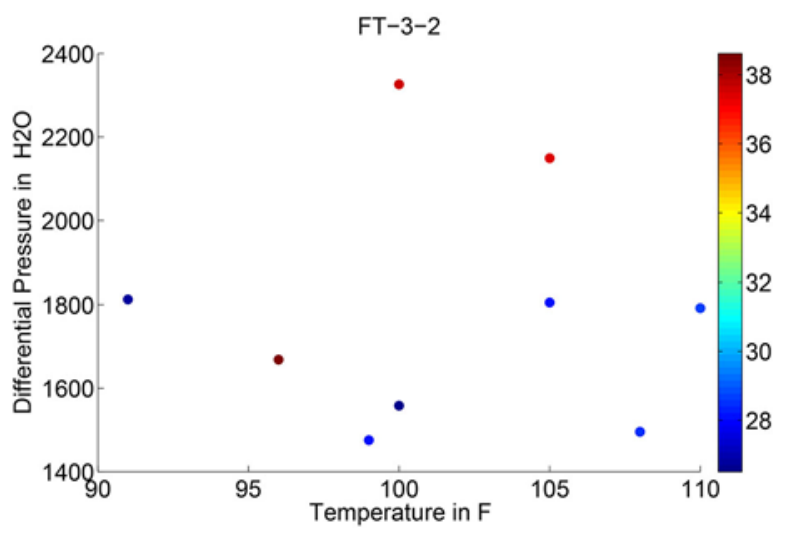

(c)

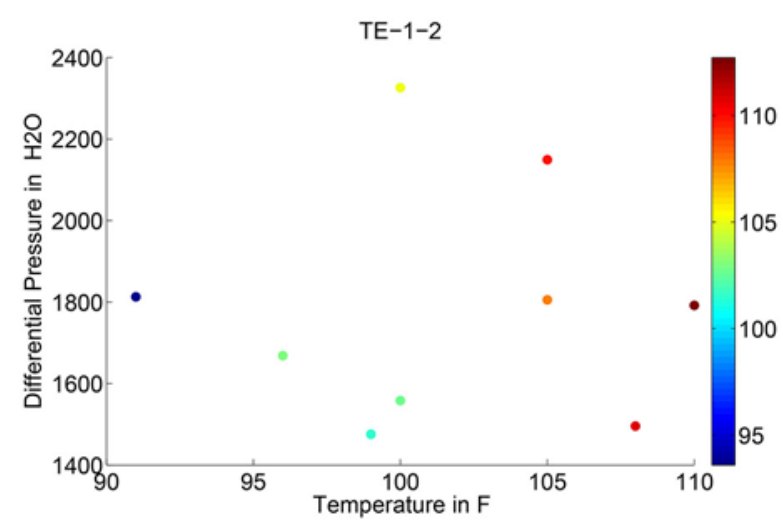

(e)

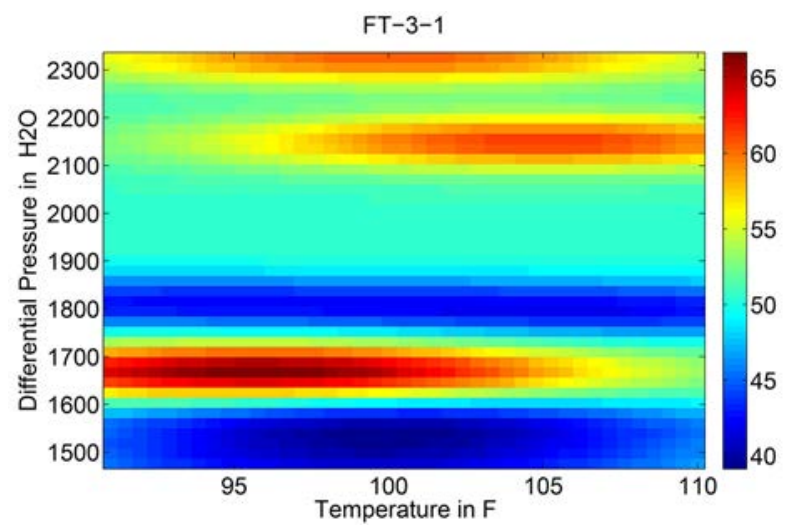

(b)

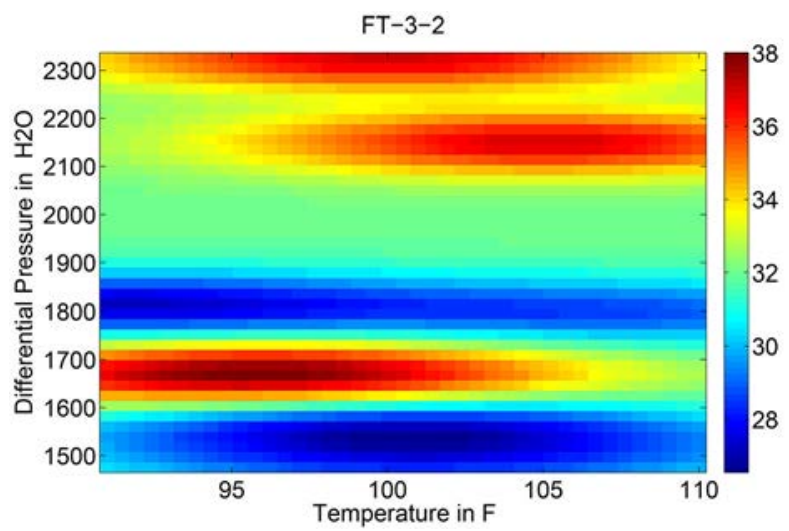

(d)

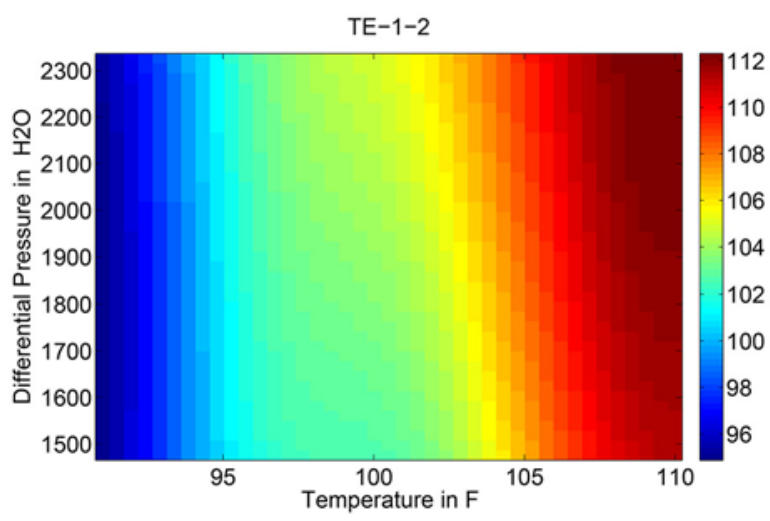

(f)

Figure 4.5. $\quad$ Predicted Response Surfaces at a Single Time Instant, Assuming Two Input Variables. (a), (c), (e), (g), (i), (k), (m), (o), (q): Observed data from each of the nine sensors used in this analysis. The horizontal axis corresponds to temperature $\left({ }^{\circ} \mathrm{F}\right)$ and the vertical axis corresponds to differential pressure (inches $\mathrm{H}_{2} \mathrm{O}$ ). Both input variables correspond to the control parameters of the instrumented flow loop. (b), (d), (f),(h),(j),(l), (n), (p), (r): Prediction surfaces (response surfaces) for the nine sensor measurements presented as a color-coded map. The response surface data only shows the prediction value and does not present the associated error bars. 


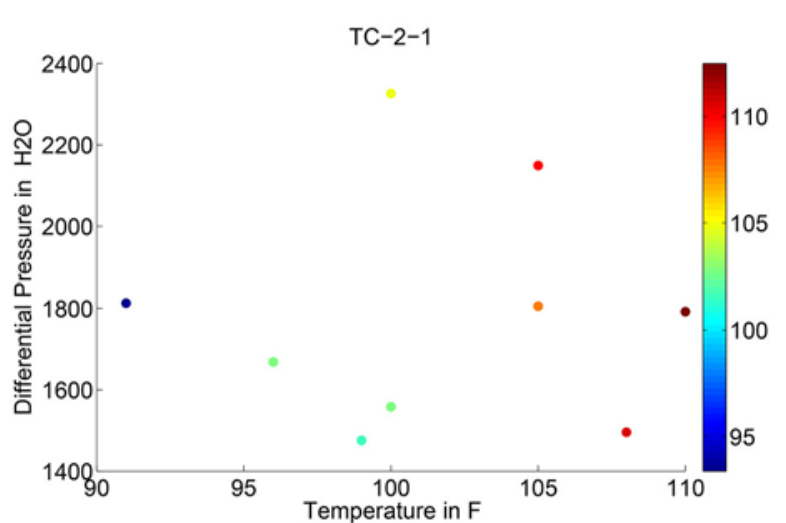

(g)

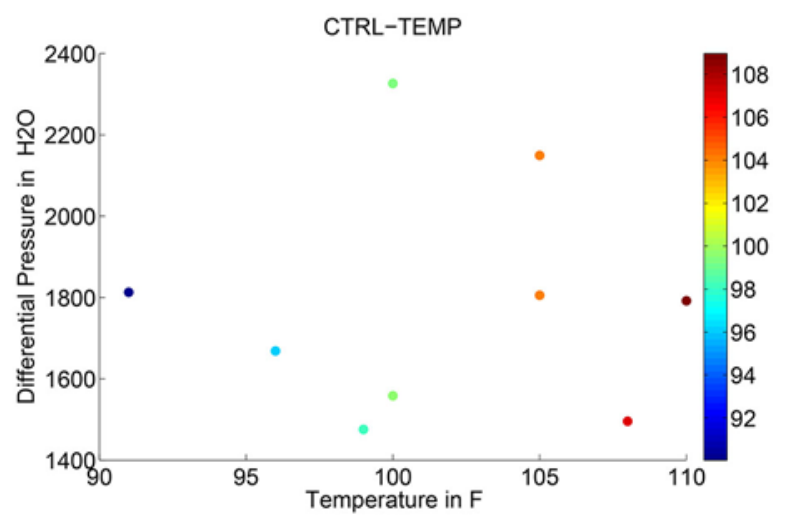

(i)

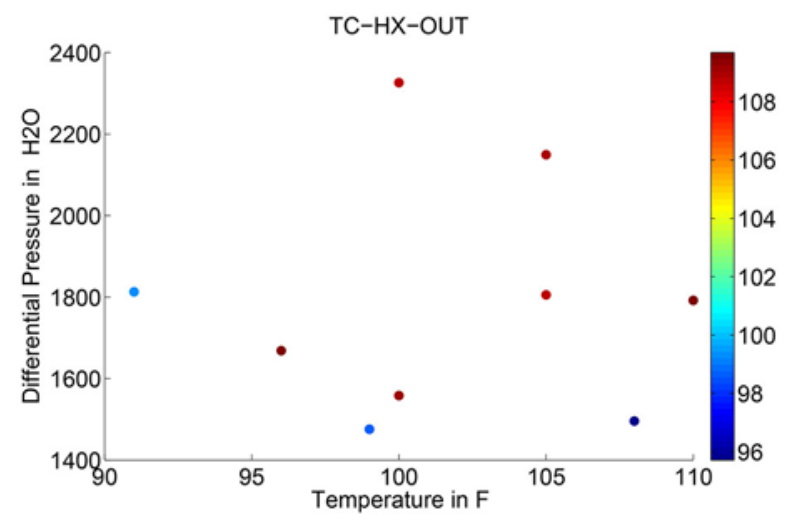

(k)

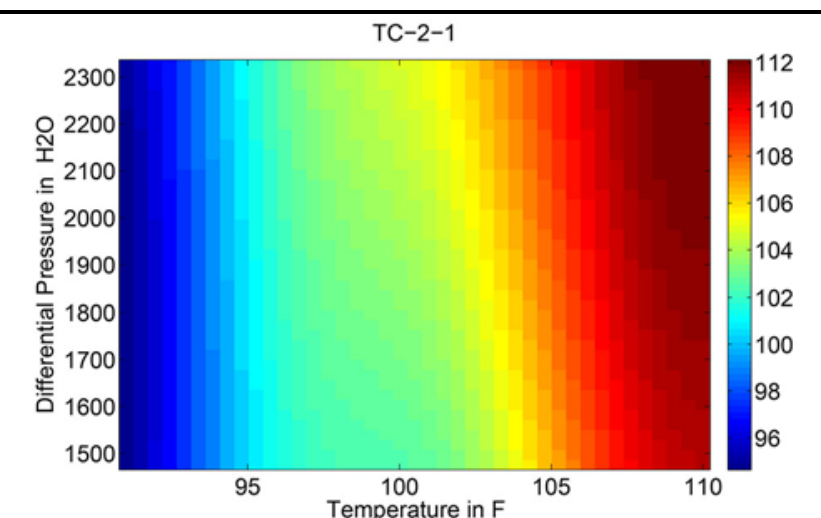

(h)

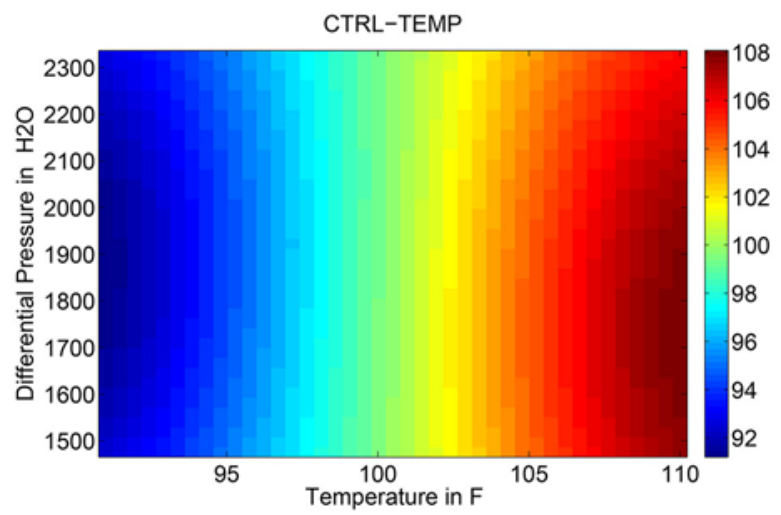

(j)

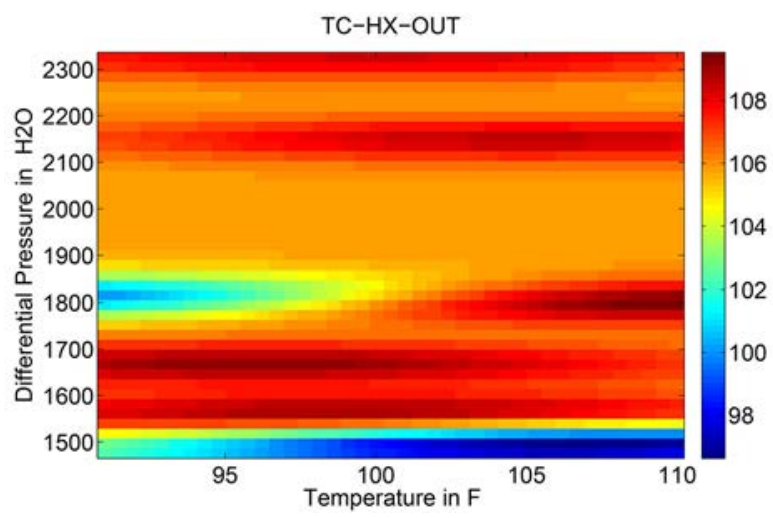

(l)

Figure 4.5. cont'd 


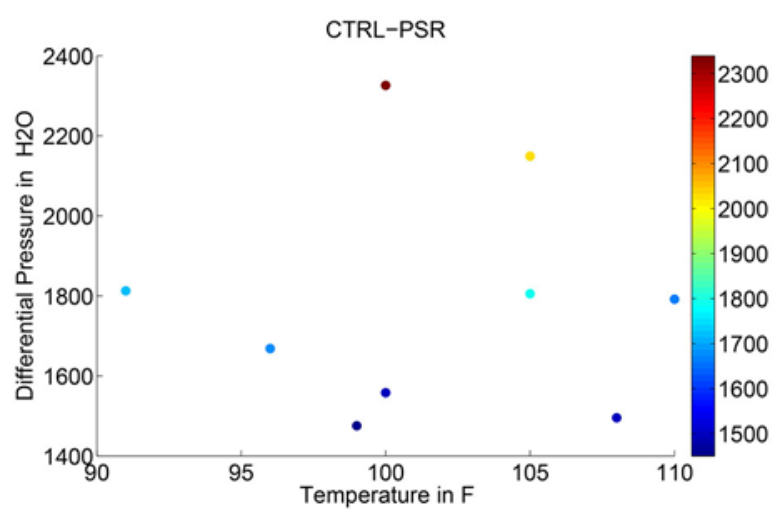

(m)

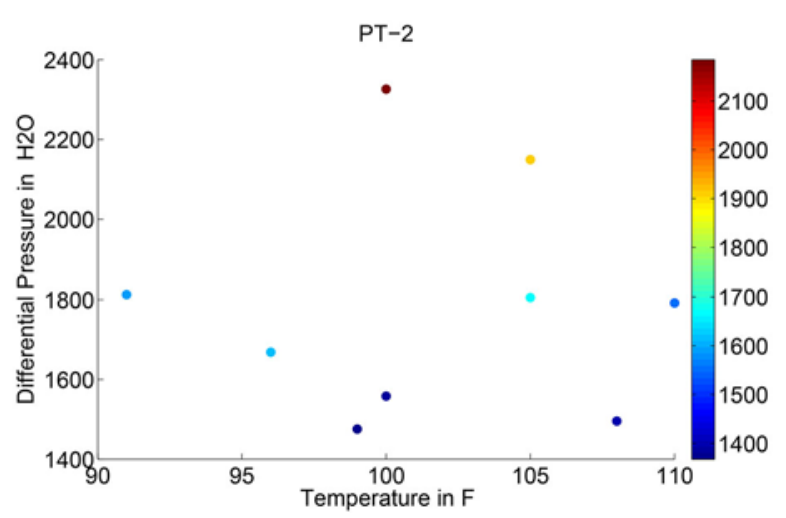

(o)

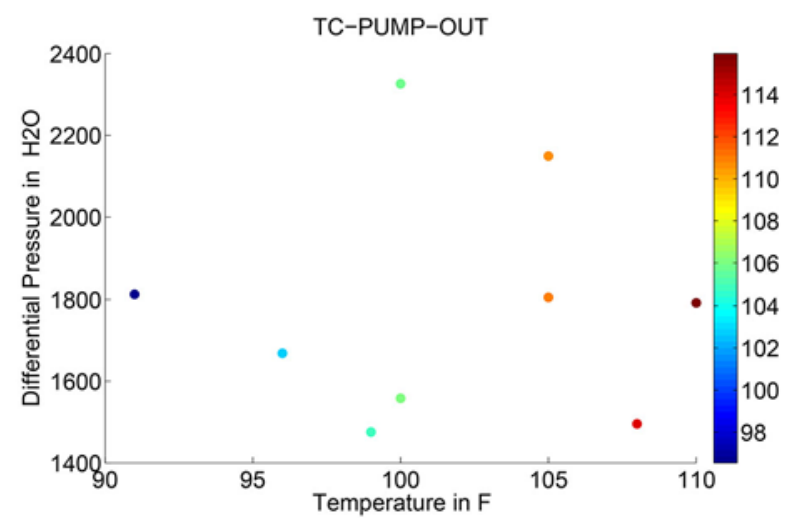

(q)

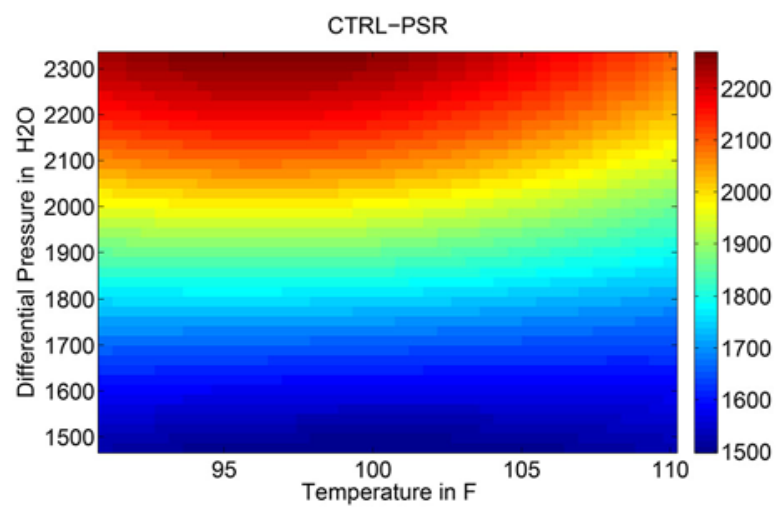

(n)

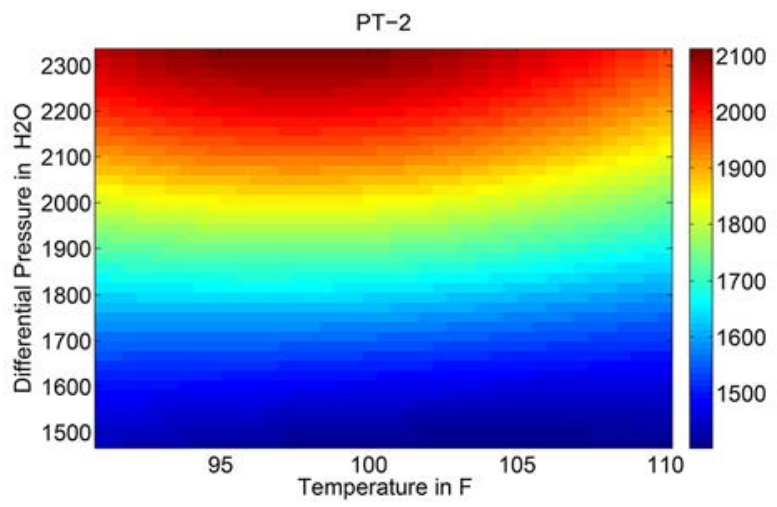

(p)

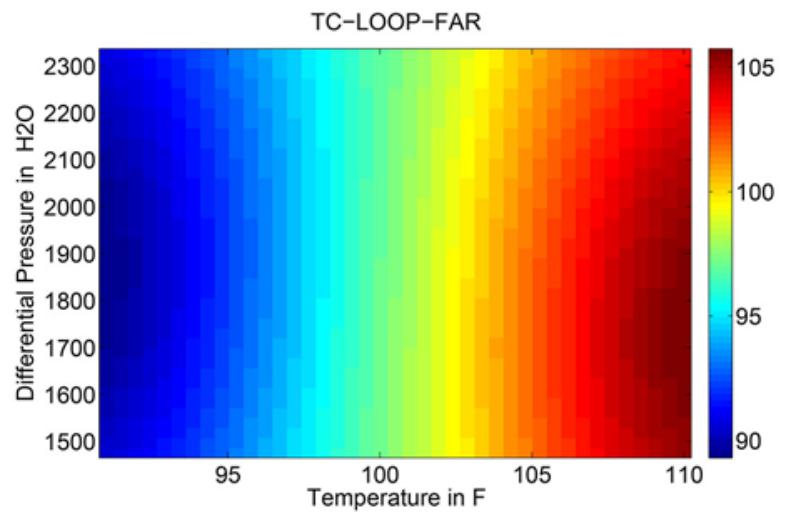

(r)

Figure 4.5. cont'd 


\subsection{Simulation Models (Flow Loop Model with Counter Flow Heat Exchanger)}

Two models of a heat exchanger flow loop were simulated in Matlab Simulink software. The first model assumed a constant flow rate in the control loop so only the temperature was controlled with a proportional integral derivative (PID) controller. The second model contained a pump, enabling the control of both the temperature and the flow rate with a controller. For both models, a concentric tube counter flow heat exchanger was represented by a series of equations. The inputs, assumptions, and outputs are listed for the constant flow model in Table 4.1.

The temperatures of the cold and hot outlet streams were calculated for given cold and hot inlet conditions, which included temperatures and mass flow rates. Within the algorithm, the heat transfer area and overall heat transfer coefficient are assumed. The specific heat of water was also used in the calculations. The calculations were based on an Effectiveness-NTU (number of transfer units) method with the total heat transfer (Q) also calculated in units of watts. Mass flow rates of the hot and cold streams were unaffected by the heat exchanger calculations so output mass flow rates were equal to the input mass flow rates.

Table 4.1. Constant Flow Model Inputs, Assumptions, and Outputs

\begin{tabular}{|c|c|c|}
\hline \multicolumn{3}{|c|}{ Inputs } \\
\hline Tc_in & Cold side inlet temperature $\left({ }^{\circ} \mathrm{C}\right)$ & {$[224]$} \\
\hline Mc_in & Cold side mass flow rate (g/s) & [3.4E6] \\
\hline Th_in & Hot side inlet temperature $\left({ }^{\circ} \mathrm{C}\right)$ & {$[300]$} \\
\hline Mh_in & Hot side flow rate (g/s) & [3.0E6] \\
\hline \multicolumn{3}{|c|}{ Assumptions } \\
\hline A & Heat transfer surface area $\left(\mathrm{m}^{2}\right)$ & \\
\hline $\mathrm{U}$ & Overall heat transfer coefficient $\left(\mathrm{W} / \mathrm{m}^{2}{ }^{\circ} \mathrm{K}\right)$ & {$[(\mathrm{U})(\mathrm{A})=1.0 \mathrm{E} 8]$} \\
\hline c & Specific heat of water & {$\left[4.186\left(\mathrm{~J} / \mathrm{g}{ }^{\circ} \mathrm{C}\right)\right.$ or $\left.\left(\mathrm{J} / \mathrm{g}{ }^{\circ} \mathrm{K}\right)\right]$} \\
\hline \multicolumn{3}{|c|}{\begin{tabular}{|c|} 
Outputs \\
\end{tabular}} \\
\hline Tc_out & Cold side outlet temperature $\left({ }^{\circ} \mathrm{C}\right)$ & [calculated output] \\
\hline Mc_out & Cold side outlet mass flow rate ( $\mathrm{g} / \mathrm{s}$ ) & [same as the input] \\
\hline Th_out & Hot side outlet temperature $\left({ }^{\circ} \mathrm{C}\right)$ & [calculated output] \\
\hline Mh_out & Hot side outlet mass flow rate (g/s) & [same as the input] \\
\hline \multicolumn{3}{|c|}{$\begin{array}{l}\text { Notes or guidelines for selected values: } \\
\text { Todreas and Kazimi (1990), Nuclear Systems I, page 418: U = 2500-50000 for boiling water and } \\
250-12000 \text { for water under forced convection in pipes. Page 5: primary coolant inlet temperature } \\
278^{\circ} \mathrm{C} \text {, average outlet temperature } 288 \text {, core flow rate } 13.1 \mathrm{Mg} / \mathrm{s} \text {. }\end{array}$} \\
\hline
\end{tabular}


The simulation was run with an Ode45 (Dormand-Prince) variable-step solver. The solver determines the time of the next step in the simulation and applies a numerical method to solve the set of ordinary differential equations representing the given model within the accuracy requirements specified. A tolerance of $1 \mathrm{E}-3$, which ensures that the computed state is accurate to within $0.1 \%$, and a run time of 800 seconds were selected.

Multiple sensors were added to a model, each one as a subsystem. A sensor subsystem was individually tailored to represent simulated noise at the sensor level. The noise levels could be matched to the type of physical sensor such as an RTD or thermocouple temperature sensor. A sensor subsystem could also represent errors such as drift, nonlinearity, and sensor bias. Furthermore, process noise was added to the constant flow model. These additions of noise to the models were selected to represent sensor and process uncertainty. A sensor subsystem is easily adapted to produce its output in either engineering units (for temperature in degrees) or sensor units (volts). The added noise was assumed to be Gaussian and was defined by a selected mean and variance.

In the constant flow model, a flow loop with a defined input set-point temperature, PID controller, and an output tracking monitor was represented as shown in Figure 4.6. The flow loop model is shown in detail in Figure 4.7 with the heat exchanger, to the right, defined by a series of equations in Matlab code. An initial input temperature on the hot side of the heat exchanger was assumed to be $300^{\circ} \mathrm{C}$ and the cold side input was fixed at $224^{\circ} \mathrm{C}$. Flow rates on the hot and cold sides were set to 3.4E6 and 3.0E6 (g/s), respectively. The full set of input parameters and assumptions are listed in Table 4.1. In-line process noise was added to the model at the point marked by the arrow in Figure 4.7. The process noise in the flow loop was defined as a Gaussian distributed random signal with 0 mean and variance of 2 . Sensor noise was added at each of the 'Record T' subsystems in the loop. The specified input set-point function and output tracking results are displayed in Figure 4.8, left and right side, respectively. There is good agreement between the output and input with evidence of the process noise. Finally the temperature over the course of the simulation at two points in the flow loop is displayed in Figure 4.9, again showing the effects of added process noise.

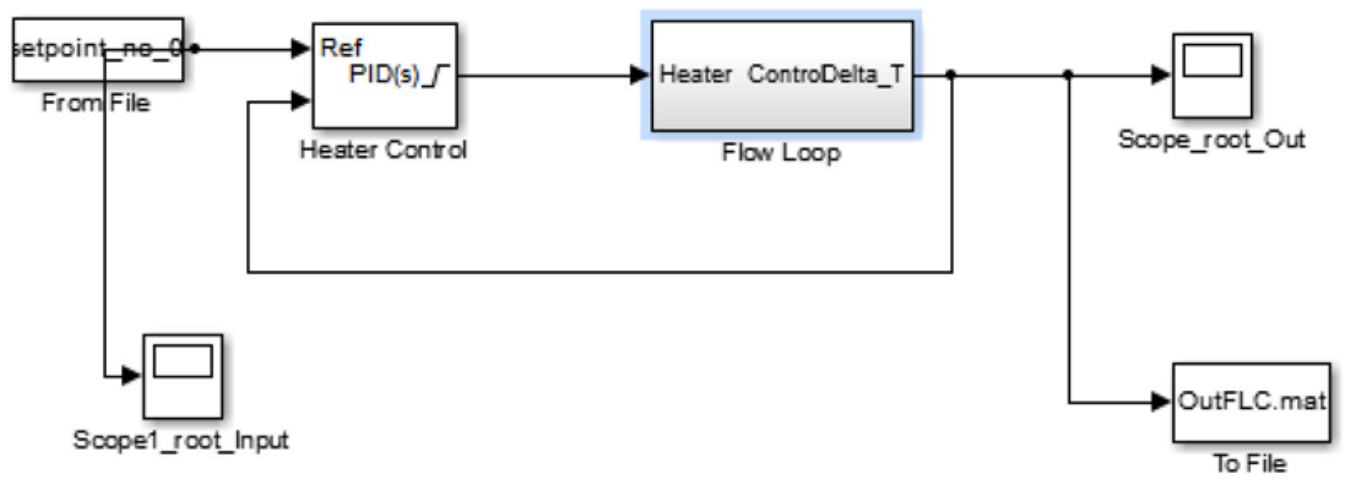

Figure 4.6. Flow Loop Control Model with Constant Flow 


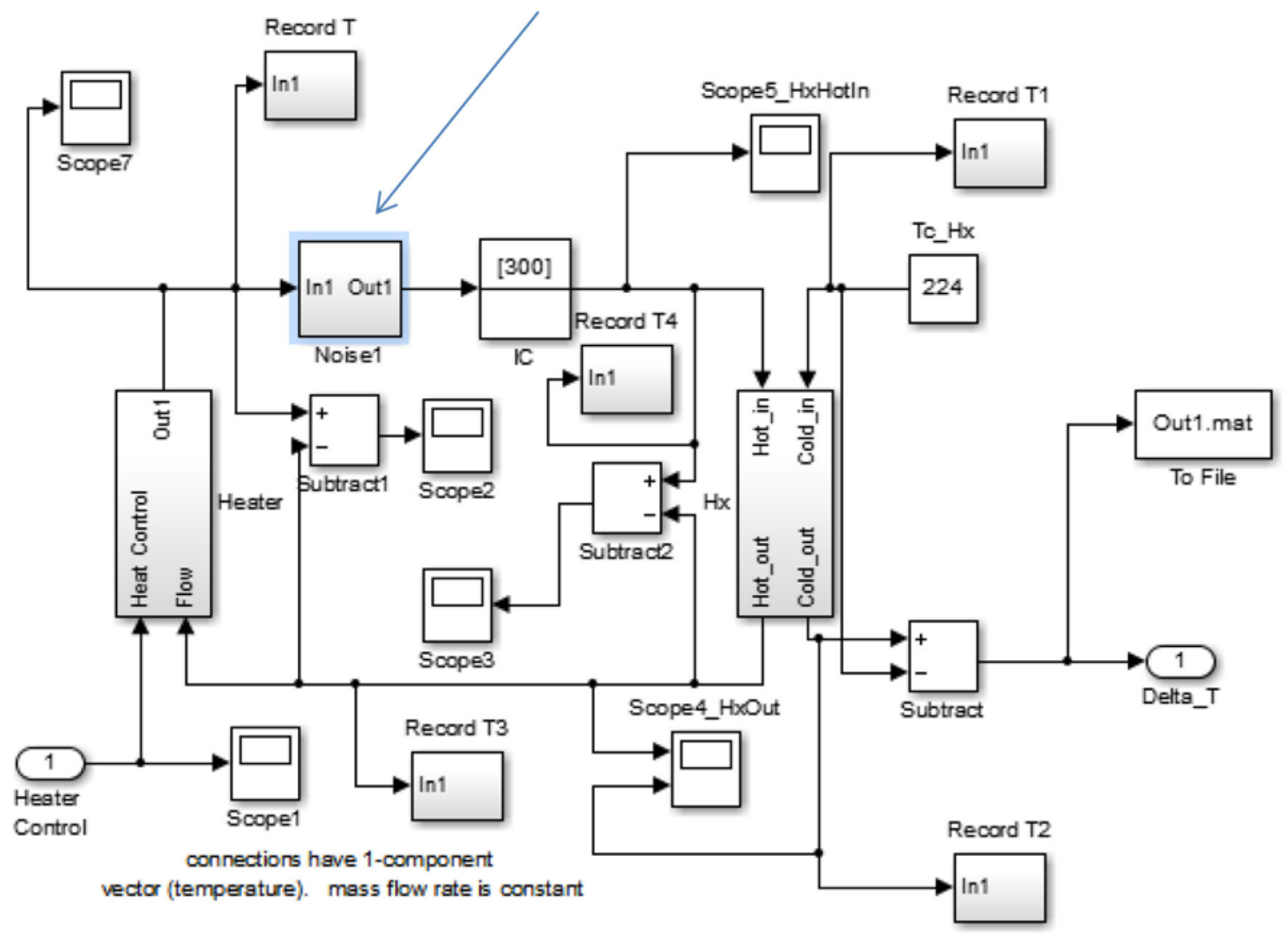

Figure 4.7. Flow Loop Details with Heater on the Left, Heat Exchanger on the Right, and Process Noise Added at the Poisition Noted by the Arrow

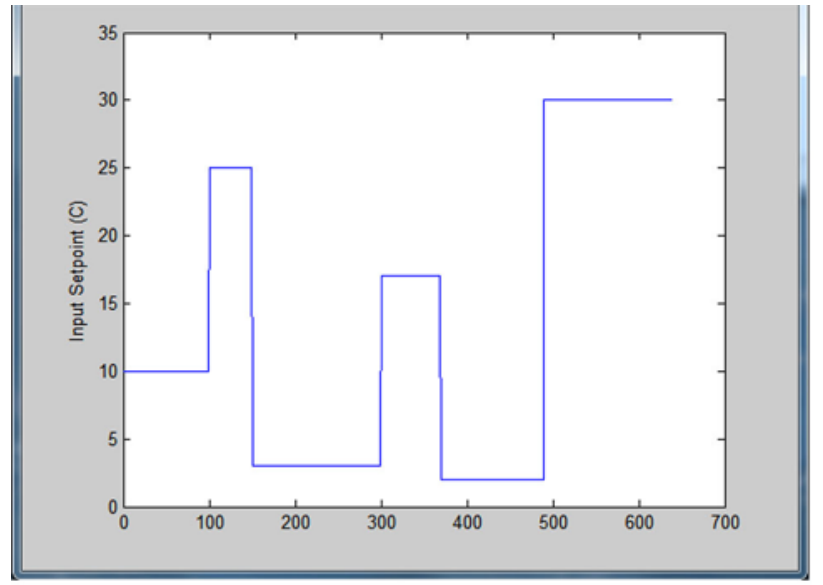

(a)

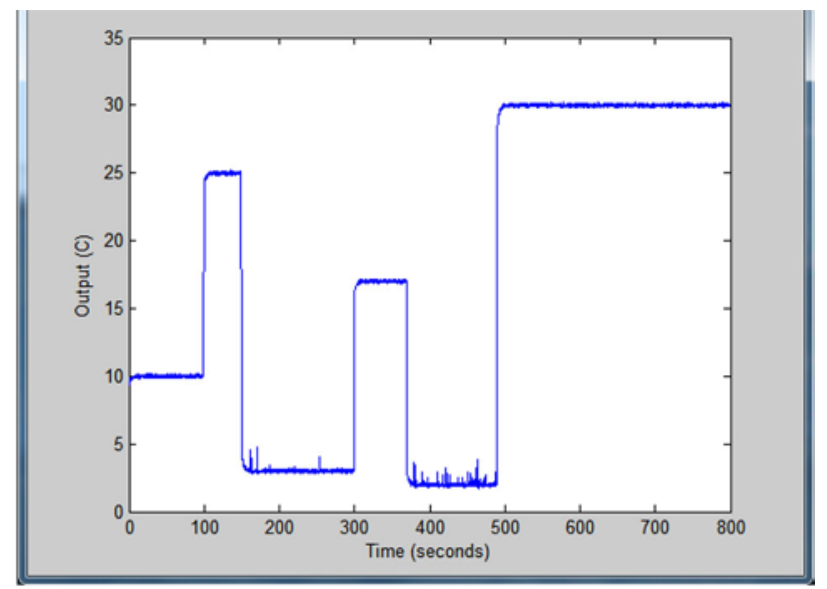

(b)

Figure 4.8. Input Setpoint Temperature on the Left and the Output Temperature on the Right 


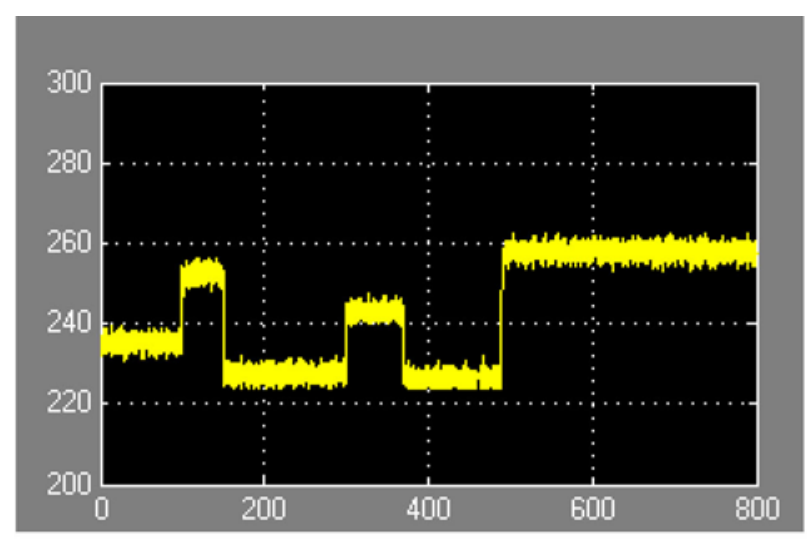

(a)

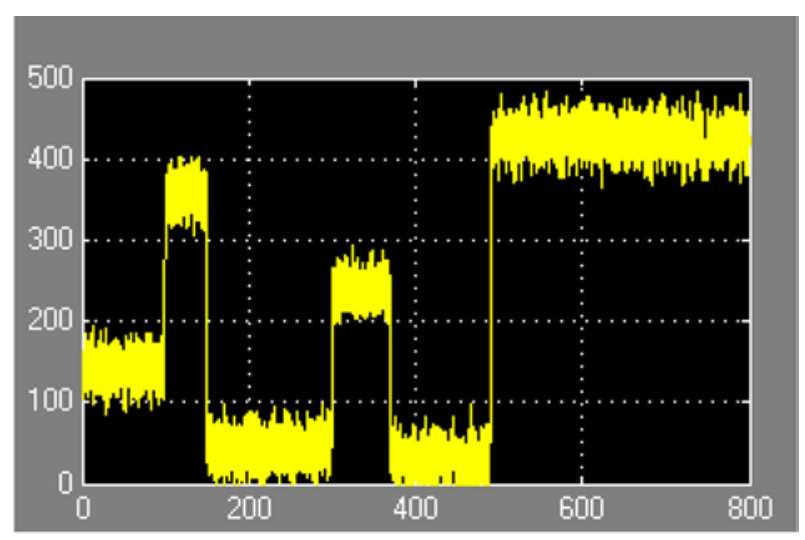

(b)

Figure 4.9. Temperatures at Different Locations in the Flow Loop, Scope 7 on the Left and Scope 1 on the Right. The horizontal axes represent time in seconds and the vertical axes represent temperature in ${ }^{\circ} \mathrm{C}$.

The variable flow model is still under development to address instabilities that occasionally lead to the model not converging or unrealistic temperatures or flow rates at some points in the loop. In general, however, the output tracked the input set-point function well. Sensor subsystems were added at various points of interest in the model to measure either temperature or flow rates and they contained sensor noise or other anomalies. The model is shown in Figure 4.10 with flow loop details shown in Figure 4.11. The same set of heat exchanger equations were used in this model as were used in the constant flow model.

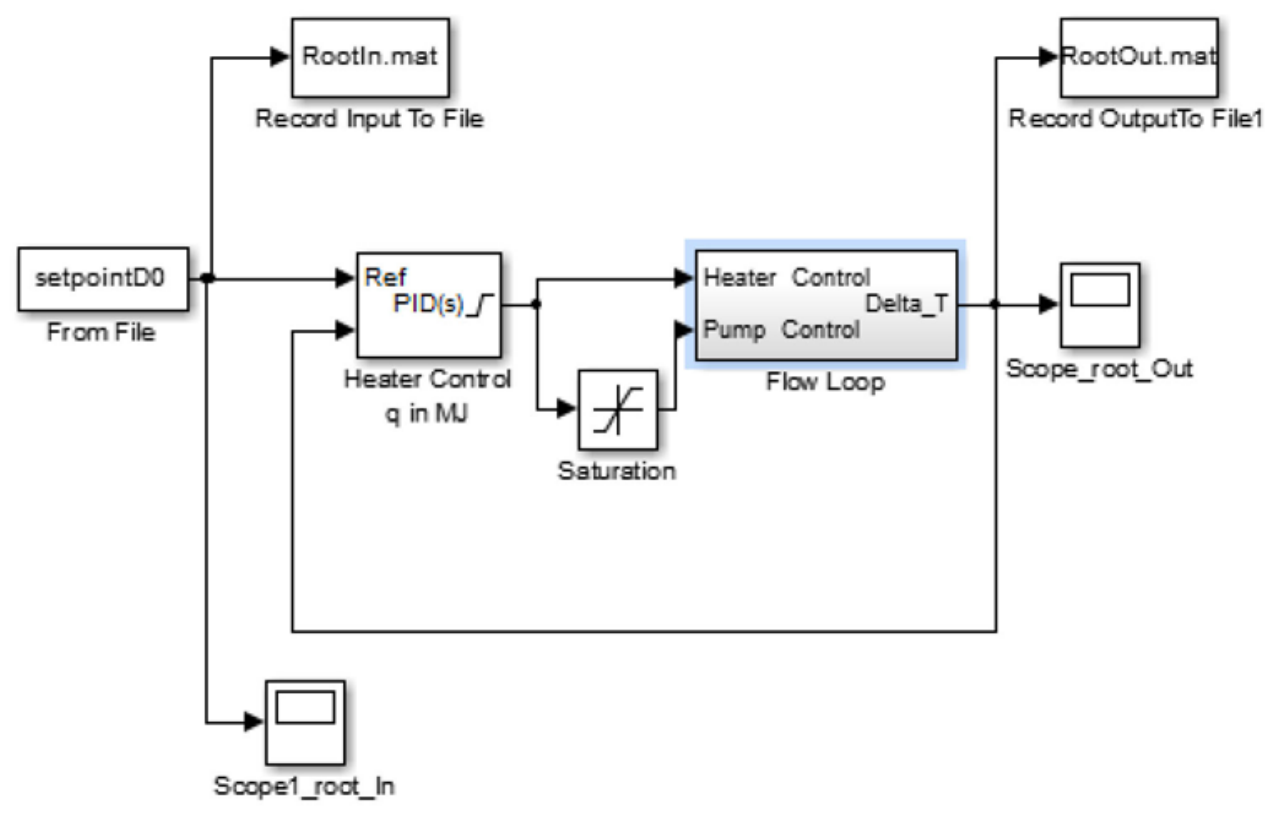

Figure 4.10. Flow Loop Model with Variable Temperature and Flow 


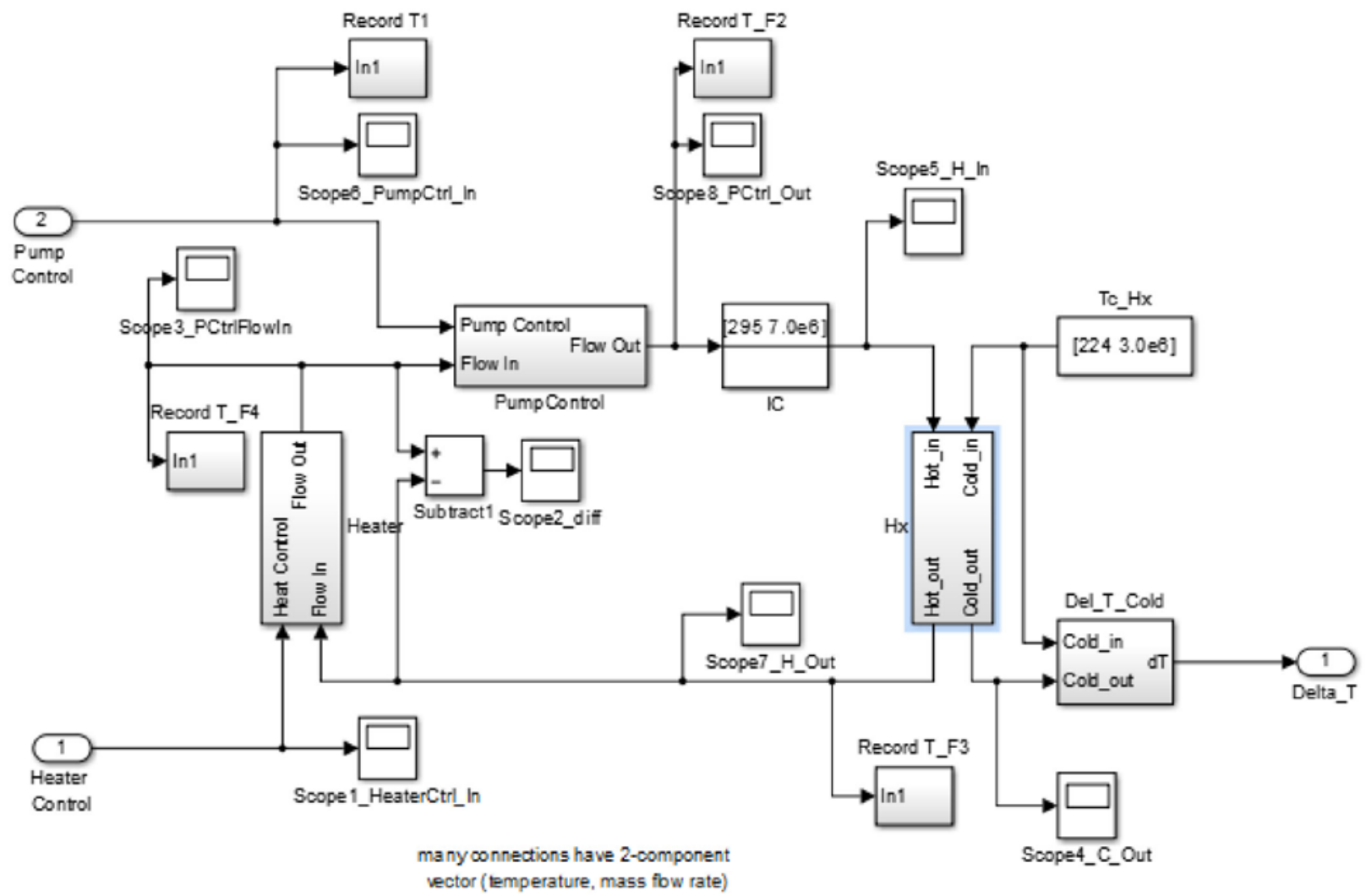

Figure 4.11. Flow Loop Details with Heater, Pump Control, Heat Exchanger, and Multiple Sensors (Record Subsystems) 


\subsection{Summary}

Periodic sensor calibration involves (1) isolating the sensor from the system, (2) applying an artificial load and recording the result, and (3) comparing this "As Found" result with the recorded "As Left" condition from the previous recalibration to evaluate the drift at several input values in the range of the sensor. If the sensor output is found to have drifted from the previous condition, then the sensor is adjusted to meet the prescribed “As Left” tolerances. Previous reviews of recalibration logs suggest that the current practice of periodic recalibration adds a significant amount of unnecessary maintenance during busy maintenance outages. Advanced algorithms that continuously monitor sensor responses can address this issue and facilitate automated monitoring and control of plant and subsystem performance.

Widespread utilization of traditional OLM approaches is lacking and a key gap that needs to be addressed to increase adoption of these technologies, particularly in the nuclear power plant arena, is the need to better quantify OLM uncertainty. Sources of uncertainty in OLM can be roughly categorized as (1) process noise, (2) measurement uncertainty, (3) electronic noise, and (4) modeling uncertainty. Approaches to UQ that are data-driven may be capable of providing estimates of uncertainty that are time-varying as the quantities being measured vary with time. Such a capability provides the option of adjusting acceptance criteria and, potentially, setpoints in a time-varying fashion to meet the needs of the nuclear power system.

A Gaussian Process model is proposed for addressing the UQ issue. The advantage of this approach is the ability to account for spatial and temporal correlations among the sensor measurements that are used in OLM. The GP model, as proposed, may be considered an extension of a commonly used OLM model and, therefore, the hypothesis is that the UQ methodology may be readily extended to accommodate commonly used OLM models.

Initial assessment of the proposed UQ methodology using data from an instrumented flow loop indicates the feasibility of generating error bounds on measurement data that are time-dependent. However, the assessment uses only steady-state data from normal conditions (i.e., no sensor faults are present) and will need to be extended to include data from sensor faults and non-steady-state conditions. These assessments are ongoing.

In parallel, simulation models of a flow loop with a counter-flow heat exchanger were developed. The simulation model serves two purposes. First, data may be generated from the simulation model that represent conditions the experimental flow loop may not be able to achieve. Second, the use of a model can provide insights into physical relationships between measurements from sensors at different locations. These insights can be used to derive new algorithms for OLM, as well as develop methods for generating virtual sensors. Evaluation of the models themselves, and analysis of data generated from the simulation models, is ongoing. Future work in this regard includes incorporating the collected measurement data parameters into the model to evaluate or validate the model results. Additionally, the variable flow model will be modified to ensure that it is a robust model of the flow loop. 



\subsection{References}

Bickford R, E Davis, R Rusaw and R Shankar. 2002. "Development of an Online Predictive Monitoring System for Power Generating Plants." In 45th ISA POWID Symposium. June 3-5, 2002, San Diego, California.

Buongiorno J. 2010. 22.06 Engineering of Nuclear Systems, Fall 2010. Massachusetts Institute of Technology. Cambridge, Massachusetts. Accessed September 18, 2013. Available at http://ocw.mit.edu/courses/nuclear-engineering/22-06-engineering-of-nuclear-systems-fall-2010. MIT OpenCourseWare: Massachusetts Institute of Technology. License: Creative Commons BY-NC-SA.

Coble JB, RM Meyer, P Ramuhalli, LJ Bond, HM Hashemian, BD Shumaker and DS Cummins. 2012. A Review of Sensor Calibration Monitoring for Calibration Interval Extension in Nuclear Power Plants. PNNL-21687, Pacific Northwest National Laboratory, Richland, Washington.

Davis E, R Bickford, P Colgan, K Nesmith, R Rusaw and R Shankar. 2002. "On-Line Monitoring at Nuclear Power Plants - Results from the EPRI On-Line Monitoring Implementation Project." In 45th ISA POWID Symposium. June 2-7, 2002, San Diego, California.

Deckert JC, JL Fisher, DB Laning and A Ray. 1983. "Signal Validation for Nuclear Power Plants." Journal of Dynamic Systems, Measurement, and Control 105:24-29.

Ding J. 2004. Independent Component Analysis for Sensor Validation. PhD Thesis, University of Tennessee, Knoxville, Knoxville, Tennessee.

EPRI. 1998. On-Line Monitoring of Instrument Channel Performance. TR 104965, Electric Power Research Institute, Palo Alto, California.

EPRI. 2000a. "Appendix J, NRC Safety Evaluation Report." In On-Line Monitoring of Instrument Channel Performance. Electric Power Research Institute, Palo Alto, California.

EPRI. 2000b. On-Line Monitoring of Instrument Channel Performance - Topical Report 104965-R1 NRC SER. EPRI, Palo Alto, CA.

EPRI. 2003. On-Line Monitoring Cost-Benefit Guide. EPRI Report 1006777, Electric Power Research Institute, Palo Alto, California.

EPRI. 2008. Requirements for On-Line Monitoring in Nuclear Power Plants. EPRI Report 1016725, Electric Power Research Institute, Palo Alto, California.

Ghanem R and P Spanos. 1991. Stochastic Finite Elements: A Spectral Approach. Springer Verlag. Reissued by Dover Publications, 2004.

Gross KC and KK Hoyer. 1993. "Reactor Parameter Signal Simulator." In Fourth International Conference on Simulation Methods in Nuclear Engineering, Volume 1. June 2-4, 1993, Montreal, Canada. Canadian Nuclear Society.

Hashemian H. 1990. Aging of Nuclear Plant Resistance Temperature Detectors. NUREG/CR-5560, U.S. Nuclear Regulatory Commission, Washington, D.C. 
Hashemian H. 1995a. On-line Testing of Calibration of Process Instrumentation Channels in Nuclear Power Plants. NUREG/CR-6343, U.S. Nuclear Regulatory Commission, Washington, D.C.

Hashemian H. 1995b. "On-line Testing of Calibration of Process Instrumentation Channels in Nuclear Power Plants (NUREG/CR-6343)."

Hashemian HM. 2006. Maintenance of Process Instrumentation in Nuclear Power Plants. Springer. ISBN 9783540337034.

Hashemian HM. 2011. "On-Line Monitoring Applications in Nuclear Power Plants." Progress in Nuclear Energy 53(2):167-181.

Hashemian HM and J Jiang. 2009. "Nuclear Plant Temperature Instrumentation." Nuclear Engineering and Design 239(12):3132-3141.

Hines JW and E Davis. 2005. "Lessons Learned from the U.S. Nuclear Power Plant On-Line Monitoring Programs." Progress in Nuclear Energy 46(3-4):176-189.

Hines JW and DR Garvey. 2006. "Development and Application of Fault Detectability Performance Metrics for Instrument Calibration Verification and Anomaly Detection." Journal of Pattern Recognition Research 1(1).

Hines JW, J Garvey, R Seibert and A Usynin. 2008a. Technical Review of On-line Monitoring Techniques for Performance Assessment, Volume 2: Theoretical Issues. NUREG/CR-6895, Vol. 2, U.S. Nuclear Regulatory Commission, Washington, D.C.

Hines JW, J Garvey, R Seibert and A Usynin. 2008b. Technical Review of On-line Monitoring Techniques for Performance Assessment, Volume 3: Limiting Case Studies. NUREG/CR-6895, Vol. 3, U.S. Nuclear Regulatory Commission, Washington, D.C.

Hines JW and B Rasmussen. 2005. "On-Line Sensor Calibration Monitoring Uncertainty Estimation." Nuclear Technology 151(3).

Hines JW and R Seibert. 2006a. Technical Review of On-line Monitoring Techniques for Performance Assessment, Volume 1: State-of-the-Art. NUREG/CR-6895, Vol. 1, U.S. Nuclear Regulatory Commission, Washington, D.C.

Hines JW and R Seibert. 2006b. "Technical Review of On-Line Monitoring Techniques for Performance Assessment: State-of-the-Art (NUREG/CR-6895, Volume 1) ". http://www.nrc.gov/reading-rm/doccollections/nuregs/contract/cr6895/v1/cr6895v1.pdf.

Hoeting JA, D Madigan, AE Raftery and CT Volinsky. 1999. "Bayesian Model Averaging: A Tutorial." Statistical Science 14(4):382-401.

IAEA. 2008. On-line Monitoring for Improving Performance of Nuclear Power Plants, Part 1:

Instrument Channel Monitoring. NP-T-1.1, International Atomic Energy Agency, Vienna, Austria.

IAEA. 2011. Core Knowledge on Instrumentation and Control Systems in Nuclear Power Plants. IAEA Nuclear Energy Series No. NP-T-3.12, International Atomic Energy Agency, Vienna. 
Ikonomopoulos A and THJJ van der Hagen. 1997. "A novel signal validation method applied to a stochastic process." Annals of Nuclear Energy 24(13):1057-1067.

ISA. 2010. Methodologies for the Determination of Setpoints for Nuclear Safety-Related Instrumentation ISA RP67.04.02:2010, International Society of Automation (ISA), Research Triangle Park, North Carolina.

Kennedy MC and A O'Hagan. 2001. "Bayesian Calibration of Computer Models." Journal of the Royal Statistical Society, Series B 63(3):425-464.

Kiureghiana AD and O Ditlevsen. 2009. "Aleatory or Epistemic? Does It Matter?" Structural Safety 31(2):105-112.

Liu W, JC Principe and S Haykin. 2010. Kernel Adaptive Filtering: A Comprehensive Introduction. Wiley. ISBN 0470447532.

Matthies HG. 2007. "Quantifying Uncertainty: Modern Computational Representation of Probability and Applications." In Extreme Man-Made and Natural Hazards in Dynamics of Structures, NATO Security through Science Series, pp. 105-135 eds: A Ibrahimbegovic and I Kozar. Springer.

Miron A. 2001. A Wavelet Approach for Development and Application of a Stochastic Parameter Simulation System. PhD Thesis, University of Cincinnati, Cincinnati, Ohio. Available at http://rave.ohiolink.edu/etdc/view?acc_num=ucin997906768.

Oh DY and HC No. 1990. "Instrument failure detection and estimation methodology for the nuclear power plant." Nuclear Science, IEEE Transactions on 37(1):21-30.

Rasmussen BP. 2003. Prediction Interval Estimation Techniques for Empirical Modeling Strategies and their Applications to Signal Validation Tasks. Ph.D. Thesis, The University of Tennessee, Knoxville.

Rasmussen CE and CKI Williams. 2006. Gaussian Processes for Machine Learning. The MIT Press, Cambridge, Massachusetts. ISBN 0-262-18253-X.

Ray A and R Luck. 1991. "An introduction to sensor signal validation in redundant measurement systems." Control Systems, IEEE 11(2):44-49.

Seibert RM, DR Garvey and JW Hines. 2006. "Prediction Intervals Versus Confidence Intervals for OnLine Sensor Monitoring." In Proceedings of the 60th Meeting of the Society for Machinery Failure Prevention Technology (MFPT), pp. 139-148. April 3-6, 2006, Virginia Beach, Virginia. Society for Machinery Failure Prevention Technology (MFPT), Haymarket, Virginia.

Stein ML. 1999. Interpolation of Spatial Data: Some Theory for Kriging. Springer.

Tamhane AC and DD Dunlop. 2000. Statistics and Data Analysis from Elementary to Intermediate. Prentice-Hall, Inc., Upper Saddle River, New Jersey.

Todreas NE and MS Kazimi. 1990. Nuclear Systems I - Thermal Hydraulic Fundamentals. Hemisphere Publishing Corporation, New York.

Wald A. 1945. "Sequential Tests of Statistical Hypotheses." The Annals of Mathematical Statistics 16(2):117-186. 
Wiener N. 1938. "The Homogeneous Chaos." American Journal of Mathematics 60(4):897-936.

Xiu D. 2010. Numerical Methods for Stochastic Computations: A Spectral Method Approach. Princeton University Press, Princeton, New Jersey. ISBN 978-0-691-14212-8. 



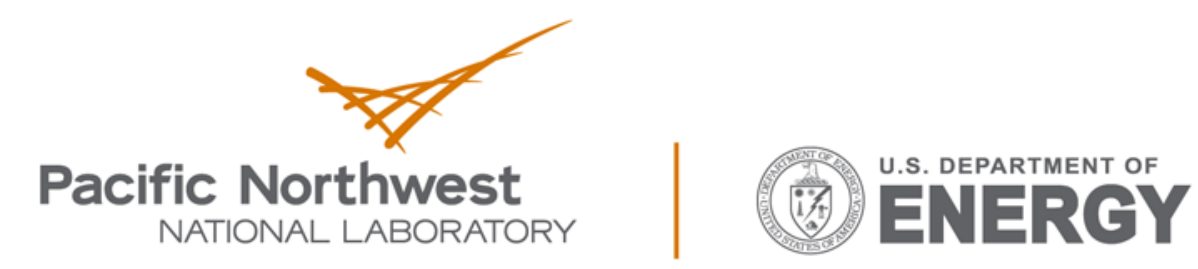

Proudly Operated by Battelle Since 1965

902 Battelle Boulevard

P.O. Box 999

Richland, WA 99352

1-888-375-PNNL (7665)

www.pnnl.gov 